

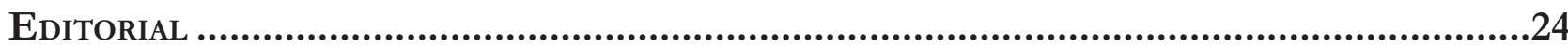

O Direito na fronteira da razão: Psicologia, neurociência e economia comportamental................... 24 Patrícia Perrone Campos Mello e Sergio Nojiri

I. NeURodireito: COGNIÇão, EMOÇÃo, JUÍZOS MORAIS E CIÊNCIA ..........................................26

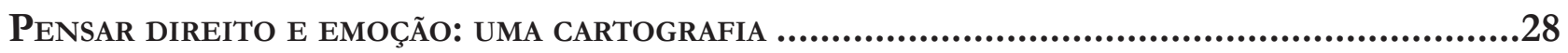

Nevita Maria Pessoa de Aquino Franca Luna

Neurodireito: o início, o fim E O MEIO

Carlos Marden e Leonardo Martins Wykrota

ENSAIO JURÍDICO SOBRE A RACIONALIDADE HUMANA: MAIORES, CAPAZES E IRRACIONAIS

André Perin Schmidt Neto e Eugênio Facchini Neto

DIVERGÊNCIAS DE PRINCÍPIO: ARGUMENTOS JURÍDICOS E MORAIS EM UM CENÁRIO DE DESACORDOS SOCIAIS

André Matos de Almeida Oliveira, Pâmela de Rezende Côrtes e Leonardo Martins Wykrota

CONSILIÊNCIA E A POSSIBILIDADE DO NEURODIREITO: DA DESCONFIANÇA À RECONCILIAÇÃO DISCIPLINAR.....

Thaís de Bessa Gontijo de Oliveira e Renato César Cardoso

MODELOS DE MORALIDADE

Molly J. Crockett

A INFELIZ BUSCA POR FELICIDADE No DiREITo

Úrsula Simões da Costa Cunha Vasconcellost, Noel Struchiner e Ivar Hannikainen

Além da liberdade: PersPeCtivas Em Nietzsche.

Lucas Costa de Oliveira

A mediaÇão de CONFlitos SOb a PERSPECTIVA do DESENVOLVIMENTO HUMANO: AS CONTRIBUIÇÕES DA PSICOLOGIA POSITIVA

Simone de Biazzi Ávila Batista da Silveira e Deise Brião Ferraz

Neuroimagiologia e aValiação de ResPonsabilidade

Nicole A. Vincent 
ANÁLISE CRÍTICA DA ORIENTAÇÃO DE CIDADÃOS COMO MÉTODO PARA OTIMIZAR DECISÕES PÚBLICAS POR MEIO DA TÉCNICA NUDGE.

Luciana Cristina Souza, Karen Tobias França Ramos e Sônia Carolina Romão Viana Perdigão

Políticas públicas e o deVer de monitoramento: “LEVANdo os Direitos A SÉrio". .252 Ana Paula de Barcellos

Nudges E POLÍticas PÚblicas: uM MECANISMO DE COMBATE AO TRABALHO EM CONDIÇÃo ANÁLOGA À DE ESCRAVO .267

Amanda Carolina Souza Silva, Débhora Renata Nunes Rodrigues e Saul Duarte Tibaldi

REDUZINDO A TRIBUTAÇÃO COGNITIVA: LIÇÕES COMPORTAMENTAIS PARA A DIMINUIÇÃO DOS EFEITOS PSICOLÓGICOS ADVERSOS DA POBREZA.............................................................288 Leandro Novais e Silva, Luiz Felipe Drummond Teixeira, Gabriel Salgueiro Soares e Otávio Augusto Andrade Santos

Políticas PÚBLICAS EM SUICÍDIO: DO PATERNALISMO CLÁSSICO AO PATERNALISMO LIBERTÁRIO E NUDGING

Davi de Paiva Costa Tangerino, Gabriel Cabral e Henrique Olive

Nudges COMO POLÍticA PÚbliCA PARA AUMENTAR O ESCASSO NÚMERO DE DOADORES DE ÓRGÃos PARA TRANSPLANTE

Roberta Marina Cioatto e Adriana de Alencar Gomes Pinheiro

Os PROGRAMAS DE INTEGRIDADE PARA CONTRATAÇÃO COM A ADMINISTRAÇÃO PÚBLICA ESTADUAL: NUDGE OU OBRIGAÇÃo LEGAL? UM OLHAR SOBRE AS DUAS PERSPECTIVAS .386

Cíntia Muniz Rebouças de Alencar Araripe e Raquel Cavalcanti Ramos Machado

Paternalismo libertário e Proteção JURídica do AMbiente: POR QUe PROTEger o AMBIENTE TAMBÉM DEVE SER PROTEGER AS LIBERDADES?

Mariana Carvalho Victor Coelho e Patryck de Araujo Ayala

Políticas PÚblicas baseadas EM EVIdÊNCIAS COMPORTAMENTAIS: REFLEXões A PARTIR do Projeto de Lei 488/2017 do Senado

Pâmela de Rezende Côrtes, André Matos de Almeida Oliveira e Fabiano Teodoro de Rezende Lara

III. ECONOMIA COMPORTAMENTAL: VIESES COGNITIVOS E POLÍTICAS PÚBLICAS .455

ECONOMIA COMPORTAMENTAL E DIREITO: A RACIONALIDADE EM MUDANÇA Marcia Carla Pereira Ribeiro e Victor Hugo Domingues

VIESES COGNITIVOS E DESENHO DE POLÍTICAS PÚBLICAS 
A neurociênCia da moralidade na tomada de DeCisões Jurídicas Complexas e No DESENHO DE POLÍTICAS PÚBLICAS

Erik Navarro Wolkart

Desvio de CARÁter ou SIMPLESMENTE HUMANO? ECONOMIA COMPORTAMENTAL APLICADA AO COMPORTAMENTO DESONESTO

Diana Orghian, Gabriel Cabral, André Pinto e Alessandra Fontana

Políticas Públicas e a ConcretizaÇão de direitos sociais: TOMAdA DE DECisão, ARQUITETURA DE ESCOLHAS E EFETIVIDADE

Ana Elizabeth Neirão Reymão e Ricardo dos Santos Caçapietra

BEHAVIORAL ECONOMICS E DIREITO DO CONSUMIDOR: NOVAS PERSPECTIVAS PARA O ENFRENTAMENTO DO SUPERENDIVIDAMENTO .568

Samir Alves Daura

A EDUCAÇÃo FORMAL PARA O CONSUMO É GARANTIA PARA UMA PRESENÇA REFLETIDA DO CONSUMIDOR NO MERCADO? UMA ANÁLISE COM BASE NA BEHAVIORAL LAW AND ECONOMICS (ECONOMIA COMPORTAMENTAL) 600

Marcia Carla Pereira Ribeiro e Edson Mitsuo Tiujo

LIBET, DETERMINISMO E CONSUMO: AS INFLUÊNCIAS DO MARKETING E A RELEVÂNCIA DA DELIBERAÇÃo CONSCIENTE NA SUPERAÇÃo CONDICIONAL DE HÁBITOS DE CONSUMO PERIGOSOS616 Émilien Vilas Boas Reis e Leonardo Cordeiro de Gusmão

CiÊNCIA DO DIREITO TRIBUTÁRIO, ECONOMIA COMPORTAMENTAL E EXTRAFISCALIDADE. .640 Hugo de Brito Machado Segundo

IV. CoMportamento JUdiCiAL: INFLUÊNCIA DE FATORES EXTRAJURÍDicos .660

FATORES METAPROCESSUAIS E SUAS INFLUÊNCIAS PARA A FORMAÇÃo DA DECISÃo JUDICIAL .662 Rogério Roberto Gonçalves de Abreu, Lúcio Grassi de Gouveia e Virgínia Colares

“A VIDA COMO ELA É": COMPORTAMENTO ESTRATÉGICO NAS CORTES Patrícia Perrone Campos Mello

A COMPOSIÇÃo do ÓRGão COLEGIAdo E SEUS EFEITOS NA TOMADA DE DECISÃo .720 André Garcia Leão Reis Valadares

Das 11 ilhas ao centro do arquipélago: os superpoderes do Presidente do STF DURANTE O RECESSO JUDICIAL E FÉRIAS .741 José Mário Wanderley Gomes Neto e Flávia Danielle Santiago Lima 
RAZÃo, EMOÇÃo E DELIBERAÇÃO: AS ADEQUAÇÕES REgIMENTAIS do SUPERIOR TribUNAL DE JUSTIÇA PARA A FORMAÇÃo DE PRECEDENTES EFICAZES

Peter Panutto e Lana Olivi Chaim

Heurística de ancoragem e fiXaÇÃo de danos morais em JUizados especiais Cíveis no Rio DE JANEIRO: UMA NOVA ANÁLISE 778

Fernando Leal e Leandro Molhano Ribeiro

LA PROTECCIÓN DE LOS DERECHOS POLÍTICOS FRENTE A LAS FUNCIONES DISCIPLINARIAS DE LAS AUTORIDADES ADMINISTRATIVAS: SUBSIDIARIEDAD Y DEFERENCIA EN EL SISTEMA INTERAMERICANO DE DERECHOS HUMANOS Jorge Ernesto Roa Roa

V. A influênCia do gÊNERo no PROCESSO DECisório JUdiCial

Como os Juízes decidem os Casos de estupro? ANALISANDo SENTENÇAS SOb A PERSPECTIVA DE VIESES E ESTEREÓTIPOS DE GÊNERO 826 Gabriela Perissinotto de Almeida e Sérgio Nojiri

GÊNERO E COMPORTAMENTO JUDICIAL NO SUPREMO TRIBUNAL FEDERAL: OS MINISTROS CONFIAM MENOS EM RELATORAS MULHERES?

Juliana Cesario Alvim Gomes, Rafaela Nogueira e Diego Werneck Arguelhes

Hércules, Hermes e a Pequena Sereia: uma reflexão sobre estereótipos de gênero, SUBPRESENTAÇÃo DAS MULHERES NOS TRIBUNAIS E (I)LEGITIMIDADE DEMOCRÁTICA DO PODER JUDICIÁRIO. .878 Jane Reis Gonçalves Pereira e Renan Medeiros de Oliveira

Prisão Cautelar de gestantes: análise do Fundamento filosófico da decisão do Habeas CoRpus N. 143.641 912

Artur César Souza e Giovania Tatibana de Souza

VI. Neurodireito APlicado ao direito E Ao Processo PENAL....................................926

CÉREbros QUe PUNEM: UMA REVISÃo CRÍTICA DA NEURoCIÊNCIA DA PUNIÇÃo .....................928 Ricardo de Lins e Horta

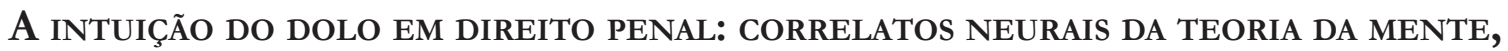
RACIOCÍNIO INDUTIVO E A GARANTIA DA CONVICÇÃO JUSTIFICADA. .946 Thiago Dias de Matos Diniz e Renato César Cardoso

As COMUNIDADES EPISTÊMICAS PENAIS E A PRODUÇÃo LEGISLATIVA EM MATÉRIA CRIMINAL..... 961 Stéphane Enguéléguélé 
DELINQUÊNCIA JUVENIL: RELAÇÕES ENTRE DESENVOLVIMENTO, FUNÇÕES EXECUTIVAS E COMPORTAMENTO SOCIAL NA ADOLESCÊNCIA .

André Vilela Komatsu, Rafaelle CS Costa e Marina Rezende Bazon

Límites TEMPORALES A LAS PENAS PRIVATIVAS DE LIBERTAD ATENDIENDO AL DESARROLLO PSICOSOCIAL.

Silvio Cuneo Nash

NEURolaw E AS PERSPECTIVAS PARA UMA ANÁLISE OBJETIVA DO COMPORTAMENTO SUGESTIONADO: REPERCUSSÃO DAS FALSAS MEMÓRIAS NA ESFERA PENAL

Mariana Dionísio de Andrade, Marina Andrade Cartaxo e Rafael Gonçalves Mota

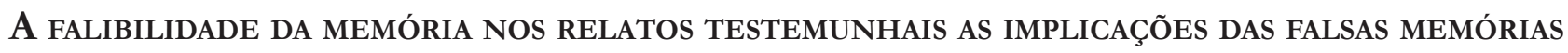
NO CONTEXTO DOS CRIMES CONTRA A DIGNIDADE SEXUAL

Caroline Navas Viana

A (IR)REPETIBILIDADE dA PROVA PENAL DEPENDENTE DA MEMÓRIA: UMA DisCUSSÃo COM BASE NA PSICOLOGIA DO TESTEMUNHO. 1058

William Weber Cecconello, Gustavo Noronha de Avila e Lilian Milnitsky Stein 


\section{Delinquência juvenil: relações entre desenvolvimento, funções executivas e comportamento social na adolescência*}

\author{
Juvenile delinquency: relationships between \\ development, executive functions, and social \\ behavior in adolescence
}

\author{
André Vilela Komatsu** \\ Rafaelle CS Costa*** \\ Marina Rezende Bazon****
}

* Recebido em 29/05/2018

Aprovado em 09/07/2018

** Doutorando em Psicologia pela Universidade de São Paulo (USP) e pesquisador visitante da Universitat de Barcelona. Membro do Grupo de Estudos e Pesquisa em Desenvolvimento e Intervenção Psicossocial (GEPDIP). Possui graduação e mestrado em Psicologia pela Universidade de São Paulo. E-mail: avk@ usp.br

*** Educadora Social do Programa de Fortalecimento de Vínculos da Organização Comunitária Santo Antônio Maria de Claret e mestranda em Psicologia pela USP. Membro do Grupo de Estudos e Pesquisa em Desenvolvimento e Intervenção Psicossocial. Possui graduação em Psicologia pela USP. E-mail: rafaellecscosta@ gmail.com

**** Docente do Departamento de Psicologia da Universidade de São Paulo (USP) e coordenadora do Grupo de Estudos e Pesquisa em Desenvolvimento e Intervenção Psicossocial. Possui graduação em Psicologia pela USP, Mestrado en Science - Psychoéducation pela Université de Montreal e Doutorado em Psicologia pela USP. E-mail: mbazon@fffclrp.usp.br

\section{Resumo}

Funções Executivas (FEs) consistem em processos mentais de ordem superior, necessários para o controle emocional, cognitivo e comportamental. A adolescência é uma etapa crucial para o desenvolvimento das FEs. Defasagens/Déficits nas funções dificultam/impossibilitam comportamentos sociais adequados e aumentam o risco para problemas de comportamento (dentre os quais a prática de delitos). O objetivo deste artigo é apresentar os resultados de uma revisão integrativa do conhecimento científico produzido em torno das relações entre FEs e comportamento delituoso na adolescência e discutir as implicações desse corpo de conhecimento reunido para programas de prevenção e tratamento/acompanhamento socioeducativo. A literatura aponta que as FEs podem se relacionar com o comportamento delituoso de forma indireta e direta. Indiretamente, disfunções executivas contribuem para a existência de dificuldades de ajustamento ao meio acadêmico e laboral, aumentando as chances de o adolescente se afastar dessas instituições sociais, de regulação da conduta, e se envolver com práticas divergentes. Diretamente, disfunções executivas, especialmente no plano do controle de impulsos, contribuem para a emissão de delitos específicos e para a violência. A literatura indica que as FEs podem ser estimuladas e desenvolvidas. Ações preventivas podem ser implementadas desde os primeiros meses de vida até à adolescência, no âmbito da família e da escola. Ações de tratamento devem focalizar as FEs mais diretamente relacionadas aos problemas manifestos e levar em consideração fatores contraproducentes ao desenvolvimento das FEs, como o estresse, o isolamento social/emocional e a saúde como um todo. Salienta-se que é necessário integrar esses conhecimentos às legislações e políticas públicas brasileiras.

Palavras-chave: Comportamento pró-social. Comportamento antissocial. Delinquência juvenil. Funções executivas. Psicologia do adolescente. 
Executive Functions (FEs) are higher order mental processes required for emotional, cognitive and behavioral control. Adolescence is a crucial stage in the development of FEs. Delays/deficits in these functions makes it impossible or more difficult to emit appropriate social behaviors and increase the risk for behavioral problems (including offenses). The objective of this study is to present the results of an integrative review of the scientific knowledge produced around the relationships between EFs and criminal behavior during adolescence and to discuss its implications for programs of prevention and treatment in socioeducative context. The literature points out that EFs relate to criminal behavior indirectly and directly. Indirectly, executive dysfunctions contribute to increase difficulties in adjusting to the academic and work environment, increasing the chances of the teenager moving away from these social institutions, which regulates the behavior, and engaging in antisocial practices. Directly, executive dysfunctions, especially regarding impulse control, contribute to the emerging of specific offenses and to violence. The literature indicates that EFs can be stimulated and developed. Preventive actions can be implemented from the first months of life through adolescence, in the family and school contexts. Treatment actions should focus on EFs specifically related to behavior problems exhibited and take into account factors counterproductive to the development of EFs, such as stress, social and emotional isolation and health as a whole. It should be noted that it is necessary to integrate this knowledge into Brazilian public policies and legislation.

Key-words: Adolescent psychology. Antisocial behavior. Executive functions. Juvenile delinquency. Prosocial behavior.

\section{INTRODUÇão}

O desenvolvimento do cérebro humano inicia-se a partir da terceira semana gestacional e continua ao longo da vida por meio de processos dinâmicos e adaptativos que promovem o surgimento e/ou a diferenciação de novas estruturas e funções — a chamada (neuroplasticidade) ${ }^{1}$. Embora essas transformações ocorram de forma contínua, alguns períodos são marcados por evidentes saltos quantitativos e qualitativos em termos de aquisições. A adolescência, estágio de transição da infância à vida adulta, é notadamente um desses períodos. Os processos neuropsicológicos que ocorrem nessa fase fazem com que a adolescência seja considerada um momento crítico para as trajetórias desenvolvimentais nos campos da cognição, das interações sociais, da personalidade e, por conseguinte, da adaptabilidade ${ }^{2}$, o que deve fomentar especial sensibilidade para com essa etapa da vida, no tocante às formas de responder às suas demandas e de lidar com as suas dificuldades, ou seja, no tocante às condições propiciadas para o crescimento e o desenvolvimento saudável dos adolescentes ${ }^{3}$, à semelhança daquela que se deve ter para com as crianças na primeira etapa da infância.

1 Os processos pelos quais o cérebro desenvolve suas estruturas e funções com base nas experiências são amplamente descritos na literatura e podem ser encontrados em: GREENWOOD, Pamela. Functional plasticity in cognitive aging: Review and hypothesis. Neuropsychology, v. 21, n. 6, p. 657-673, 2007; PAUS, Tomás; KESHAVAN, Matcheri; GIEDD, Jay. Why do many psychiatric disorders emerge during adolescence? Nature Reviews Neuroscience, v. 9, n. 12, p. 947-957, 2008; STILES, Joan; JERNIGAN, Terry. The Basics of Brain Development. Neuropsychology Review, v. 20, n. 4, p. 327-348, 2010.

2 Devido a condições peculiares do desenvolvimento do cérebro durante a adolescência, as experiências vividas nessa fase produzem efeitos mais duradouros que experiências vividas posteriormente, conforme indicam os estudos: ARAIN, Mariam et al. Maturation of the adolescent brain. Neuropsycbiatric Disease and Treatment, v. 9, p. 449-461, 2013; PAUS, Tomás; KESHAVAN, Matcheri; GIEDD, Jay. Why do many psychiatric disorders emerge during adolescence? Nature Reviews Neuroscience, v. 9, n. 12, p. 947-957, 2008. 3 A criança e o adolescente, por serem indivíduos em desenvolvimento, necessitam de proteção e de cuidado especial, devendo ser amparados pela família, sociedade e Estado com o objetivo de se propiciar um ambiente favorável ao desenvolvimento integral e harmônico de sua personalidade: LIMA, Renata Mantovani de; POLI, Leonardo Macedo; JOSÉ, Fernanda São. A Evolução Histórica dos Direitos da Criança e do Adolescente: da insignificância jurídica e social ao reconhecimento de direitos e garantias fundamentais. Rev. Bras. Polít. Públicas, Brasília, v. 7, n. 2, p. 313-329, 2017. 
As diversas oscilações emocionais e comportamentais observadas na adolescência podem ser atribuídas, ao menos em parte, a um descompasso natural no estágio desenvolvimental entre diferentes áreas cerebrais e, consequentemente, suas respectivas funções. Por exemplo, os circuitos neuronais ligados ao sistema de recompensas se estabelecem mais rapidamente que os circuitos ligados ao controle comportamental e emocional e ao de planejamento de longo prazo, o que tornam os jovens mais propensos a buscarem atividades de risco, e menos inclinados a planejarem seu futuro ${ }^{45}$. Essa condição, somada a determinadas condições sociais e culturais, algumas específicas à adolescência, fazem com que essa fase seja a de maior incidência de diferentes problemas de comportamento — internalizantes e externalizantes —, bem como de muitos transtornos mentais ${ }^{678}$.

Embora todos os adolescentes passem pelos mesmos processos maturacionais, o tempo em que eles ocorrem variam de indivíduo para indivíduo, e são influenciados por diversos fatores como: genética; níveis dos hormônios sexuais estrógeno, progesterona e testosterona; qualidade nutricional; padrões de sono; estresse físico ou psicológico; abuso de drogas e outros estímulos ambientais ${ }^{9}$. Esses determinantes do desenvolvimento neural fazem com que cada indivíduo possua padrões singulares de ligações estruturais e funcionais, denominados conectoma (termo que pode ser compreendido como uma espécie de impressão digital neural). $\mathrm{O}$ conectoma indica a aptidão de cada indivíduo para desenvolver competências relacionadas às funções executivas $(\mathrm{FEs})^{10}{ }^{11}$.

Função Executiva - FE (também conhecida como controle executivo ou controle cognitivo) é um amplo construto que remete a processos cognitivos de ordem superior que governam comportamentos guiados por objetivos e respostas adaptativas a situações novas, complexas ou ambíguas ${ }^{12}$. Em relação às FEs, há consenso geral de que existem três núcleos básicos: Controle Inibitório; Memória de Trabalho; Flexibilidade Cognitiva. Desses núcleos derivam uma série de subfunções necessárias à autorregulação emocional e comportamental apropriada à execução de tarefas cotidianas ${ }^{13}$. Diferenças individuais na performance dessas funções são preditores e moderadores relevantes para uma série de resultados na vida, como sucesso acadêmico, laboral e relacional ${ }^{14} 15$.

O Controle Inibitório refere-se à capacidade de o indivíduo controlar a própria atenção, emoção, pensamento e comportamento, o que lhe permite escolher ou mudar como reagir ou comportar-se frente aos estímulos ambientais ou aos impulsos/às predisposições internas ${ }^{16}$. As funções relacionadas a esse núcleo podem ser divididas em controle de interferência (atenção seletiva e inibição cognitiva) e autocontrole (ini-

4 ARAIN, Mariam et al. Maturation of the adolescent brain. Neuropsycbiatric Disease and Treatment, v. 9, p. 449-461, 2013.

5 KONRAD, Kerstin; FIRK, Christine; UHLHAAS, Peter. Brain Development During Adolescence: Neuroscientific Insights Into This Developmental Period. Deutsches Ärzteblatt International, v. 110, n. 25, p. 425-431, 2013.

6 LEE, Francis et al. Adolescent mental health-Opportunity and obligation: Emerging neuroscience offers hope for treatments. Science, New York, v. 346, n. 6209, p. 547-549, 2014.

7 MOFFITT, Terrie. Male antisocial behaviour in adolescence and beyond. Nature Human Behaviour, v. 2, n. 3, p. 177-186, 2018.

8 PAUS, Tomás; KESHAVAN, Matcheri; GIEDD, Jay. Why do many psychiatric disorders emerge during adolescence? Nature Reviews Neuroscience, v. 9, n. 12, p. 947-957, 2008.

9 Alguns estudos revisaram os principais determinantes do desenvolvimento neural e como eles afetam à saúde física e mental do indivíduo: ARAIN, Mariam et al. Maturation of the adolescent brain. Neuropsychiatric Disease and Treatment, v. 9, p. 449-461, 2013; e GALVÁN, Adriana. Adolescence, brain maturation and mental health. Nature Neuroscience, v. 20, n. 4, p. 503-504, 2017.

10 FINN, Emily et al. Functional connectome fingerprinting: Identifying individuals using patterns of brain connectivity. Nature Neuroscience, v. 18, n. 11, p. 1664-1671, 2015.

11 KAUFMANN, Tobias et al. Delayed stabilization and individualization in connectome development are related to psychiatric disorders. Nature Neuroscience, v. 20, n. 4, p. 513-515, 2017.

12 HUGHES, Claire; GRAHAM, Andrew; GRAYSON, Andrew. Executive function in childhood: Development and disorder. In: OATES, John. (Ed.). Cognitive Development. Oxford: Open University Press, 2005. p. 205-230.

13 DIAMOND, Adele. Executive functions. Annual Review of Psychology, v. 64, p. 135-168, 2013.

14 DIAMOND, Adele. Executive functions. Annual Review of Psychology, v. 64, p. 135-168, 2013.

15 ROMERO, María. Executive control enhancement and cognitive training. 2017. Tese (Doutorado), Universidad de Granada, Granada, 2017.

16 DIAMOND, Adele. Executive functions. Annual Review of Psychology, v. 64, p. 135-168, 2013. 
bição comportamental e emocional). No tocante ao controle de interferência, a atenção seletiva capacita o indivíduo a focar estímulos de seu interesse e suprimir a atenção dos demais estímulos ${ }^{17}$. A inibição cognitiva permite a supressão de representações mentais como pensamentos ou memórias indesejáveis ou irrelevantes para determinada situação, o que tem grande influência sobre a memória de trabalho ${ }^{18}$. Essas duas classes de habilidades são fundamentais para o aprendizado e o sucesso acadêmico.

A segunda dimensão do controle inibitório, o autocontrole, envolve a capacidade de exercer controle sobre as próprias emoções e comportamentos, resistindo a vontades ou a prazeres imediatos em detrimento de metas de longo prazo, mais significativas ${ }^{19}{ }^{20}$. Vale ressaltar que, embora para vários autores o conceito de autocontrole seja mais amplo ${ }^{21} 22$, abrangendo quase todos os aspectos dos três núcleos de FEs mencionados anteriormente, na classificação de Diamond ${ }^{24}$, embasada em investigações relacionados a substratos neurais, o termo autocontrole se restringe às dimensões emocional e comportamental.

Memória de Trabalho, outro núcleo das FEs, compreende a capacidade de manter, temporariamente, e de manipular informações na mente com o objetivo de executar tarefas cognitivas simples e complexas como compreensão de linguagem, aprendizado e raciocínio ${ }^{25}$. Entre outras funções, a memória de trabalho é responsável por incorporar novas informações ao pensamento, transformar instruções em planos de ação, considerar outros pontos de vista, relacionar ideias semelhantes e separar ideias distintas ${ }^{26}$. Essas habilidades são fundamentais para uma série de tarefas do dia a dia.

Com relação ao terceiro núcleo básico das FEs, a Flexibilidade Cognitiva, nesse as funções se desenvolvem como desdobramento do desenvolvimento das funções nos dois núcleos anteriores; elas remetem à capacidade de ajustar apropriadamente o próprio comportamento frente às mudanças ambientais ${ }^{27}$. A flexibilidade cognitiva nos permite mudar nosso foco de atenção de um estímulo para outro; atualizar nossas crenças com base em novas informações; considerar múltiplos elementos simultaneamente; "quebrar" pensamentos complexos em unidades mais simples; considerar perspectivas diferentes em um mesmo cenário ${ }^{28}$ ${ }^{29}{ }^{30}$. Essas habilidades também são fundamentais para uma série de tarefas do dia a dia.

As evidências científicas revelam que todas essas funções, relacionadas a estruturas neurais, estão em franco desenvolvimento durante a adolescência e que, mesmo indivíduos da mesma idade, podem diferir bastante em relação ao seu ritmo maturacional. Um atraso desenvolvimental na aquisição dessas funções pode ter origem em fatores biológicos e/ou ambientais e impossibilita ou eleva o custo de respostas apropriadas/esperadas em determinados contextos. Assim, levando em consideração que pressões e exigências sociais se impõem aos indivíduos tendo, de forma geral, como parâmetro, sua idade cronológica, muitos

17 DIAMOND, Adele. Executive functions. Annual Review of Psychology, v. 64, p. 135-168, 2013.

18 NOREEN, Saima; MACLEOD, Malcolm. What Do We Really Know about Cognitive Inhibition? Task Demands and Inhibitory Effects across a Range of Memory and Behavioural Tasks. PLoS ONE, v. 10, n. 8, 2015.

19 DELISI, Matt. Low Self-Control Is a Brain-Based Disorder. In: BEAVER, Kevin; BARNES, JC; BOUTWELL, Brian. The nurture versus biosocial debate in criminology: on the origins of criminal behavior and criminality. Thousand Oaks: Sage Publication Inc., 2014.

20 DIAMOND, Adele. Executive functions. Annual Review of Psychology, v. 64, p. 135-168, 2013.

21 DUCKWORTH, Angela; KERN, Margaret. A meta-analysis of the convergent validity of self-control measures. Journal of Research in Personality, v. 45, n. 3, p. 259-268, 2011.

22 FUJITA, Kentaro. On conceptualizing self-control as more than the effortful inhibition of impulses. Personality and Social Psychology Review, v. 15, n. 4, p. 352-366, 2011

23 GOTTFREDSON, Michael; HIRSCHI, Travis. A general theory of crime. Stanford, CA: Stanford University Press, 1990.

24 DIAMOND, Adele. Executive functions. Annual Review of Psychology, v. 64, p. 135-168, 2013.

25 BADDELEY, Alan. Working memory. Science, v. 255, n. 5044, p. 556-9, 1992.

26 DIAMOND, Adele. Executive functions. Annual Review of Psychology, v. 64, p. 135-168, 2013.

27 DIAMOND, Adele. Executive functions. Annual Review of Psychology, v. 64, p. 135-168, 2013.

28 DAJANI, Dina; UDDIN, Lucina. Demystifying cognitive flexibility: Implications for clinical and developmental neuroscience. Trends in Neurosciences, v. 38, n. 9, p. 571-578, 2015.

29 DIAMOND, Adele. Executive functions. Annual Review of Psychology, v. 64, p. 135-168, 2013.

30 HAUSER, Tobias et al. Cognitive flexibility in adolescence: Neural and behavioral mechanisms of reward prediction error processing in adaptive decision making during development. Neuroimage, v. 104, p. 347-354, 2015. 
adolescentes se encontram em situações em que não são capazes de responder conforme as expectativas sociais, independentemente de suas vontades ou esforços. O problema é que, por essa razão, muitos são submetidos a respostas ambientais também inadequadas/rígidas, que tendem a reiterar as dificuldades desses adolescentes, no plano das aquisições relacionadas às FEs.

A manifestação de comportamentos de risco, incluindo práticas desviantes, passíveis de serem tipificadas como atos infracionais, na adolescência, pode ser compreendida nessa perspectiva. Sabe-se que comportamentos delituosos, nessa etapa da vida, são comuns e, certamente, se atrelam aos processos desenvolvimentais em curso ${ }^{31}$. Na perspectiva da Criminologia Desenvolvimental, a grande questão é entender por que, para a maioria dos adolescentes, esses comportamentos tendem a ser manifestações pontuais, ao passo que, para um subgrupo, se tornam repetitivos, denotando um padrão de funcionamento (a)típico, que risca contaminar, negativamente, a trajetória desenvolvimental do indivíduo. Nessa corrente, investigações foram realizadas e, em relação às variáveis pessoais relacionadas, reiteradamente identificadas, compreendem-se as FEs como preditoras ou como moderadoras.

No âmbito do Controle Inibitório, defasagens/déficits nas funções inerentes ao controle de interferência (atenção seletiva e inibição cognitiva) e ao autocontrole (inibição comportamental e emocional), predizem delinquência juvenil, pois estas remetem a habilidades fundamentais para o aprendizado e o sucesso acadêmico ${ }^{32}$. Déficits/defasagens no autocontrole relacionam-se, ainda, com a delinquência juvenil de outras formas. Primeiramente, de modo indireto, tem-se que baixo autocontrole contribui para uma série de resultados negativos na vida, devido à propensão para falhar em engajar-se em planos de vida de mais longo prazo, como estudos, emprego e relacionamentos, o que favorece a adoção de um estilo de vida desviante ${ }^{33}$ 34 35. De modo direto, baixo autocontrole, enquanto indicador de impulsividade e desregulação emocional, concorre para a busca por satisfação imediata às próprias e/ou para reações violentas mediante frustrações, o que aumenta a suscetibilidade para ações criminosas ${ }^{36}{ }^{37}$.

No tocante à Memória de Trabalho, considerando-se o fato de as funções inerentes serem fundamentais na avaliação do risco envolvido em determinadas atividades, tem-se que defasagens/déficits nessas, de modo direto, aumentam a probabilidade de uso abusivo de $\operatorname{drogas}^{38}$ e de envolvimento em atividades delituosas ${ }^{39}$. Ademais, as defasagens / os déficits afetam o processamento de informações sociais, recurso cognitivo responsável por decodificar a intenção atribuída aos outros (hostil versus não hostil, por exemplo), na interação social, o que, também, contribui para a emissão de comportamentos violentos ${ }^{40}{ }^{41}$.

31 MOFFITT, Terrie. Male antisocial behaviour in adolescence and beyond. Nature Human Behaviour, v. 2, n. 3, p. 177-186, 2018.

32 BAZON, Marina; SILVA, Jorge; FERRARI, Renata. Trajetórias escolares de adolescents em conflito com a lei. Educação em Revista, Belo Horizonte, v. 29, n. 02, p. 175-199, 2013.

33 DELISI, Matt. Low Self-Control Is a Brain-Based Disorder. In: BEAVER, Kevin; BARNES, JC; BOUTWELL, Brian. The nurture versus biosocial debate in criminology: on the origins of criminal behavior and criminality. Thousand Oaks: Sage Publication Inc., 2014.

34 FARRINGTON, David et al. Criminal Careers Up to Age 50 and Life Success Up to Age 48: New Findings from the Cambridge Study in Delinquent Development. PsycEXTRA Dataset, 2006.

35 KOMATSU, André Vilela; BAZON, Marina Rezende. Adolescentes em conflito com a lei: justiça juvenil pela perspectiva da criminologia desenvolvimental. In: NOJIRI, S. (Org.). Direito, Psicologia e Neurociência. Ribeirão preto: IELD, 2016. 186p.

36 KOMATSU, André Vilela; BAZON, Marina Rezende. Personal Differences among Brazilian Adolescents with Distinct Levels of Engagement in Delinquency. International Journal of Criminology and Sociology, v. 6, p. 65-74, 2017.

37 WALTERS, Glenn. Low Self-Control, Peer Rejection, Reactive Criminal Thinking, and Delinquent Peer Associations: Connecting the Pieces of the Crime Puzzle. Journal of Developmental and Life-Course Criminology, v. 2, n. 2, p. 209-231, 2016.

38 PANWAR, Karni et al. Differential associations between impulsivity and risk-taking and brain activations underlying working memory in adolescents. Addictive Behaviors, v. 39, n. 11, p. 1606-1621, 2014.

39 NORDVALL, Olov; JONSSON, Bert; NEELY, Anna. Self-reported and performance-based measures of executive functions in interned youth. Psychology, Crime \& Law, v. 23, n. 3, p. 240-253, 2016.

40 FRANCO, Mariana; BAZON, Marina. Social Information Processing and Aggravation of Conduct in Young Offenders. International Annals of Criminology, v. 55, n. 01, p. 114-131, 2017.

41 MEIJERS, Jesse et al. Differences in executive functioning between violent and non-violent offenders. Psychological Medicine, v. 47, n. 10, p. 1784-1793, 2017. 
Com relação à Flexibilidade Cognitiva, defasagens/déficits predizem comportamentos delituosos e/ou violentos ${ }^{42}{ }^{43}$ porque, segundo alguns autores ${ }^{44}{ }^{45}$, estas/estes remetem a dificuldades em incorporar ideias morais e éticas abstratas nos raciocínios que subsidiam as ações sociais. Assim, indivíduos com atraso em funções de flexibilidade cognitivas acabam ajustando seus comportamentos às suas próprias demandas, especialmente sob condições estressantes, porque têm dificuldade em considerar outras perspectivas ou em captar sinais sociais, o que pode contribuir para ações ou reações inadequadas, criminais e violentas ${ }^{46}$.

Visando entender melhor como as defasagens / os déficits neurodesenvolvimentais se produzem e se relacionam com a delinquência juvenil, com vistas à consideração dessas informações na construção e na implementação de políticas públicas para a juventude, especificamente na área da Justiça Juvenil, o objetivo deste artigo é apresentar os resultados de uma revisão integrativa do conhecimento científico produzido em torno das funções executivas no que respeita ao comportamento delituoso na adolescência e discutir as implicações desse conhecimento em termos de intervenção de prevenção e de tratamento/acompanhamento socioeducativo.

\section{Método}

Há um crescente interesse em estudar a relação entre FEs e comportamento delituoso, principalmente com o advento de novos métodos e instrumentos nos campos da neurociência e da avaliação neuropsicológica. A título de exemplo, o cruzamento dos termos "executive function" com os termos "delinquen*", "offen*" ou "crime*" na base de dados Web of Science resulta em quatro produções até o ano 1999; 37 produções de 2000 a 2010; e 99 produções de 2011 até maio de 2018. Esse montante de pesquisas, embora não possa, ainda, ser considerado volumoso, já produziu um conhecimento significativo na área. Esse é, em parte, apresentado neste artigo, de forma integrada, com vistas a sumarizar os estudos levantados e estabelecer uma conclusão sobre o tema investigado. Para isso, os artigos mais recentes do indexador Web of Science (literatura internacional) e da biblioteca eletrônica Scielo Brasil (literatura nacional), dos últimos 10 anos, foram selecionados com base nos descritores supramencionados, privilegiando aqueles com métodos — instrumentos e amostras - distintos entre si, visando ilustrar as diversas formas pelas quais o objeto de investigação foi abordado e fornecer um panorama mais atual e completo possível, sem ser repetitivo. Todos os artigos selecionados têm amostra composta por adolescentes em conflito com a lei. Para melhor controlar o efeito de variáveis intervenientes, foram excluídos estudos que se propuseram a investigar adolescentes em situação de vulnerabilidade social (sem histórico de cometimento de delitos), com diagnóstico de desordem psiquiátrica e/ou com comorbidades relacionadas.

\section{Funções Executivas e Comportamentos Delituosos}

Destaca-se, primeiramente, que a grande maioria dos estudos recuperados lançou mão de testes neuropsicológicos, de base psicométrica, para avaliar diferentes aspectos das FEs. Alguns autores sublinham que os testes não devem ser considerados a medida padrão ouro das FEs e que, portanto, devem ser utilizados

42 PIHET, Sandrine et al. Cognitive and Emotional Deficits Associated with Minor and Serious Delinquency in High-Risk Adolescents. Psychiatry, Psychology and Law, v. 19, n. 3, p. 427-438, 2012.

43 VILÂ-BALLÓ, Adrià et al. Neurophysiological correlates of cognitive flexibility and feedback processing in violent juvenile offenders. Brain Research, v. 1610, p. 98-109, 2015.

44 JEVTIC, Bisera. Moral Judgement of Delinquents. Procedia: Social and Behavioral Sciences, v. 149, p. 449-455, 2014.

45 PONTIUS, Anneliese. Neurological aspects in some type of delinquency, especially among juveniles. Toward a neurological model of ethical action. Adolescence, v. 7, p. 289-308, 1972.

46 MILLER, Laurence. Neuropsychological perspectives on delinquency. Behavioral Sciences \& the Law, v. 6, p. 409-428, 1988. 
em conjunto com outras formas de coleta de dados, principalmente as observações e os crivos/as escalas de pontuação, em contextos naturais da vida do indivíduo ${ }^{47}$. Afirmam, contudo, que o método de testagem tem valor por ser rápido, econômico e não invasivo, o que viabiliza ou facilita muitas atividades, tanto de pesquisa, quanto das instituições e dos profissionais que prestam serviço de avaliação neuropsicológica. Nesses casos, defasagens em FEs podem ser detectadas com base na medida do desempenho com relação aos seguintes aspectos: tempo para a iniciação da tarefa; qualidade do planejamento e do organização do comportamento; capacidade para inibir impulsos e/ou alterar o foco da atenção e/ou em reter informações relevantes para a execução da tarefa; capacidade para corrigir os próprios erros ou usar feedbacks para mudar estratégias que não funcionam ${ }^{48}$. Assim, serão descritos e discutidos estudos que tiveram como objetivo investigar a relação entre o desempenho em FEs e o comportamento delituoso, e o fizeram por meio de testes neuropsicológicos.

Interessantemente, num dos primeiros estudos identificados, os autores Enns, Reddon, Das e Boudreau ${ }^{49}$ investigaram a existência de déficits executivos em adolescentes infratoras do sexo feminino $(\mathrm{n}=$ 100), por meio do instrumento Cognitive Assessment System (CAS), composto por um conjunto de 12 subtestes que avaliam planejamento, atenção, processamento simultâneo e processamento sucessivo. As adolescentes obtiveram desempenhos inferiores à média da população em todas as tarefas. Foi verificado maior tamanho de efeito entre o processamento sucessivo das adolescentes infratoras e não infratoras, seguidos de tamanhos de efeito médios em planejamento e na pontuação geral do CAS. Enns e colegas ${ }^{50}$ sugerem, assim, que alguns déficits neuropsicológicos específicos podem diferenciar grupos de adolescentes infratoras de não infratoras, a exemplo do que outros estudos verificariam em meio a adolescentes do sexo masculino.

No estudo de Syngelaki, Moore, Savage, Fairchild e Van Goozen ${ }^{51}$, por exemplo, diversos domínios de FEs foram investigados em adolescentes infratores do sexo masculino ( $\mathrm{n}=103$ ), comparando-os com um grupo controle $(\mathrm{n}=84)$, por meio da aplicação dos seguintes instrumentos: Wisconsin Card Sorting Test $\left(\right.$ WCST $^{52}$ - um dos testes mais usados para avaliar FEs, em específico a Habilidade de Atenção Alternada; Cambridge Neuropsychological Test Automated Battery (CANTAB), uma bateria de testes computadorizada que avalia Memória de Trabalho Espacial, Atenção Alternada e Aprendizagem Reversa, e Habilidade de Planejamento Espacial; Decision-making task (versão modificada do The Risky Choice Task - RCT) ${ }^{53}$, teste computadorizado que avalia o comportamento de tomada de risco por meio de um jogo de apostas com recompensas diferenciais. Os resultados obtidos pelo WCST indicaram maior número de erros perseverativos (que denotam insistência em um padrão de resposta, mesmo quando ele não é mais recompensado) no grupo dos adolescentes infratores, sugerindo dificuldade em alterar o próprio comportamento. A partir dos testes do CANTAB, verificou-se que os infratores cometeram mais erros nas tarefas de memória de trabalho espacial e apresentaram menos habilidades de planejamento de tarefas e para mudar o próprio comportamento.

47 BARKLEY, Russell. Executive Functions: What they are, How they work, and Why they evolve. New York: The Guilford Press, 2012.

48 STRAUSS, Esther; SHERMAN, Elisabeth; SPREEN, Otfried. A compendium of neuropsychological tests: administration, norms, and commentary. 3. ed. New York: Oxford University Press, 2016.

49 ENNS, Richard; REDDON, John; DAS, JP; BOUDREAU, Allison. Measuring executive functions in female delinquents using the Cognitive assessment system. Journal of Offender Rehabilitation, v. 47, n. 1/2, p. 3-23, 2008.

50 ENNS, Richard; REDDON, John; DAS, JP; BOUDREAU, Allison. Measuring executive functions in female delinquents using the Cognitive assessment system. Journal of Offender Rehabilitation, v. 47, n. 1/2, p. 3-23, 2008.

51 SYNGELAKI, Eva et al. Executive Functioning and risky decision making in Young male offenders. Criminal Justice and Behavior, v. 36, n. 11, p. 1213-1227. 2009.

52 O WCST é composto por cartões com diferentes formas geométricas (estrela, triângulo, círculo), em diferentes cores e quantidades. O participante deve escolher um critério para associar um cartão a outro e, após isso, ele recebe um feedback (o aplicador informa ao participante se a associação está correta ou não). Após completar uma sequência correta, a regra do teste muda (os cartões passam a ser associados de outra forma) e o feedback do aplicador ao participante também muda. Assim, o participante deve se adaptar cognitivamente a essas mudanças.

53 No RCT, o participante é apresentado a duas rodas da fortuna na tela do computador e deve escolher qual das duas proporcionará maior chance de acerto. Sem que o participante saiba, as rodas proporcionam probabilidades diferentes de ganho de pontos, assim como recompensas diferentes. 
Referente à capacidade de tomar decisões aferida pelo RCT, verificou-se maior propensão dos infratores para apostas e escolhas mais arriscadas, sobretudo depois de receber uma recompensa pequena. Observou-se, contudo, que frente a recompensas muito grandes ou muito pequenas os adolescentes infratores e não infratores se comportaram de modo semelhante.

Os autores argumentam que dificuldades em relação à aprendizagem reversa e à modulação do próprio comportamento (quando alterada a dinâmica de recompensa) e os prejuízos de memória de trabalho são consistentes com a literatura da área. A regulação do próprio comportamento e a aprendizagem reversa — com base em alterações nas demandas do ambiente — são fundamentais para o processo de controle inibitório que, por sua vez, tem importância para a capacidade de abdicar à prática de delitos, frente a oportunidades; a memória de trabalho, em seu turno, relacionar-se-ia mais com a possibilidade do adolescente ter sucesso em atividades pró-sociais, que demandam habilidades dessa natureza.

Syngelaki e colegas ${ }^{54}$, todavia, declaram que esperavam que os adolescentes do estudo formassem uma amostra heterogênea em termos de dificuldades emocionais e comportamentais, visto que, na amostra, provavelmente, havia adolescentes com padrões diferenciados de comportamento delituoso. $\mathrm{O}$ início da prática de delitos tardio (na segunda metade da adolescência) prediz um padrão que, em tese, remete a uma trajetória de implicação em delitos menos grave, geralmente menos associada a defasagens psicossociais, se comparada a trajetórias cuja prática de delitos tem início precoce (no final da infância/no início da adolescência), as quais, em geral, remetem a uma implicação em delitos mais grave, persistente ${ }^{55}$.

Levando-se em consideração esses aspectos, Muscatello e colegas ${ }^{56}$ se propuseram a estudar as FEs especificamente em adolescentes infratores $(n=147)$ de início tardio. Para tanto, utilizaram os seguintes instrumentos: WCST; Teste de Fluência Verbal ${ }^{57}$, um teste de produção verbal que avalia controle inibitório, memória de trabalho, velocidade de processamento e habilidade de planejamento; Teste de Stroop ${ }^{58}$, que contém estímulos visuais e verbais conflitantes e avalia controle inibitório e flexibilidade cognitiva. Além disso, utilizaram o Teste de Matrizes Progressivas de Raven ${ }^{59}$, que proporciona medida de funcionamento intelectual global (QI). Os infratores do estudo apresentaram desempenhos de produção na faixa clínica aquém do normativo - na tarefa de Fluência Verbal e baixa performance no WCST, ambos sinalizando prejuízo em flexibilidade cognitiva. Ademais, necessitarem de mais tempo que o esperado para responder à fase de interferência de atenção no Teste de Stroop, o que denota dificuldades no processo de controle inibitório. Ainda, identificou-se QI inferior à média.

Déficits dessa ordem não eram esperados em adolescentes cujo comportamento delituoso teria tido início na segunda metade da adolescência, remetendo a episódios mais circunscritos. Esses dados em aparente contradição com a literatura levam a pensar sobre a importância de se considerar sob quais condições os déficits executivos representam um fator implicado na manifestação do comportamento delituoso. Os

54 SYNGELAKI, Eva et al. Executive Functioning and risky decision making in Young male offenders. Criminal Justice and Behavior, v. 36, n. 11, p. 1213-1227. 2009.

55 FARRINGTON, David et al. Criminal Careers Up to Age 50 and Life Success Up to Age 48: New Findings from the Cambridge Study in Delinquent Development. PsycEXTRA Dataset, 2006.

56 MUSCATELLO, Maria et al. Executive functions and basic symptoms in adolescent antisocial behavior: A cross-sectional study on an Italian sample of late-onset offenders. Comprehensive Psychiatry, v. 55, p. 631-638, 2014.

57 No Teste de Fluência Verbal, o participante é convidado a falar, em um minuto, todas as palavras que se recorda com a letra F; depois, com a letra A e, em seguida, com a letra S. Por fim, também em um minuto, deve falar todos os nomes de animais que se recordar.

58 O Teste de Stroop é composto por três cartões de fundo branco; o Cartão A contém retângulos de diferentes cores, frente aos quais o participante deve verbalizar as cores correspondentes; o Cartão B contém palavras neutras escritas nas mesmas cores dos retângulos do Cartão A, e neste cartão o participante deve dizer os nomes das cores das palavras; O último, o Cartão C, é composto por palavras que nomeiam cores, impressas em cores diferentes, conflitantes (exemplo: a palavra "verde" escrita na cor azul), diante das quais o participante é orientado a responder da mesma forma como fez no cartão anterior, verificando as cores das palavras. $\mathrm{O}$ participante é avaliado pelo número de erros e pelo tempo de latência para completar a tarefa do Cartão C.

59 O teste é composto por 12 conjuntos de itens, dos quais o participante deve escolher uma figura entre as possíveis para completar um padrão para cada item. 
autores postularam que os déficits denotados nas FEs nesses adolescentes representariam um fator de risco para a continuidade do comportamento delituoso.

Nesse sentido Borrani, Frías, Ortiz, Garcia e Valdez ${ }^{60}$ conduziram uma investigação com objetivo semelhante aos das já descritas, lançando mão do Teste de Stroop como ferramenta de avaliação. Considerando o fato de a maior parte dos infratores apresentarem defasagem escolar, os pesquisadores, contudo, formaram grupos de adolescentes não infratores, para comparação, tendo por critério a apresentação ou não defasagem escolar. Assim, trabalharam com um grupo de adolescentes não infratores com defasagem escolar ( $\mathrm{n}=$ 27) e com outro sem defasagem escolar $(n=27)$, e com um de adolescentes infratores $(n=27)$. A justificativa refere-se ao fato de que a escolaridade tende a gerar melhores desempenhos.

Os resultados revelaram que, para o domínio de inibição comportamental, os grupos de adolescentes com defasagem escolar (infratores e não infratores) apresentram performance semelhante entre si e pior que os adolescentes não infratores com escolaridade compatível à idade, sugerindo que a baixa performance pode estar mais associada à escolaridade que ao comportamento delituoso. Para o domínio de flexibilidade cognitiva, o desempenho dos três grupos foi semelhante. Os domínios investigados não diferenciaram, portanto, adolescentes infratores de não infratores com baixa escolarização.

Os resultados de Borrani e colegas ${ }^{61}$ são interessantes à medida em que convidam à consideração de outros fatores - não discutidos/investigados — que podem proteger um grupo de se tornar infrator ou aumentar o risco para que outro grupo se torne. Contudo, ele não permite conclusões definitivas. Estas dependem de avaliações mais completas, que apreendam o funcionamento das funções neuropsicológicas em toda a sua complexidade. Neste estudo, utilizou-se, apenas, um teste. Conforme indica a revisão de Moffitt e Caspi ${ }^{6}$, nem todos os adolescentes com déficits executivos cometerão delitos porque há outras variáveis mediadoras, que podem influenciar esse comportamento. Por isso, as avaliações nesse campo devem ser realizadas e interpretadas com parcimônia, considerando-se tantos fatores quanto possível, de forma a não fomentar conclusões indevidas ${ }^{63}$.

Focalizando estudos mais específicos, atinentes à relação entre FEs e delitos de natureza específica, Miyaguchi e Shirataki ${ }^{64}$ compararam grupos de adolescentes infratores com históricos de delitos sexuais ( $\mathrm{n}$ = 55) e sem histórico de delitos sexuais $(\mathrm{n}=155)$ por meio dos seguintes instrumentos: Behavioural Assessment of the Dysexecutive Syndrome - BADS, uma bateria de seis testes que avalia déficits executivos; Wechsler Adult Intelligence Scale - Third Edition (WAIS-III; Wechsler, 1997); Wechsler Intelligence Scale for Children - Third Edition (WISC-III; Wechsler, 1991), que remete a escalas compostas por 14 subtestes que aferem o funcionamento intelectual global do adolescente e resultam em quocientes de inteligência (QI) Geral, Verbal e de Execução. Os autores controlaram a variável QI, que sabidamente é um fator que pode interferir nos resultados, identificando infratores sexuais com baixo QI $(\mathrm{n}=27)$ e com QI normativo $(\mathrm{n}=28)$, e infratores não sexuais com baixo QI $(n=105)$ e com QI normativo $(n=50)$. A partir da comparação entre os grupos de baixo QI, observou-se que os escores dos adolescentes com histórico de delitos sexuais foi significativamente menor que os dos adolescentes sem histórico de delitos sexuais no escore geral da BADS e em dois de seus subtestes: Rule Shift Cards Test (que avalia atenção alternada) e Modified Six Elements (que avalia memória prospectiva, que pode ser concebida como lembrar-se de fazer uma ação no futuro, que é um aspecto da

60 BORRANI, Jorge et al. Analysis of Cognitive inhibition and flexibility in juvenile delinquentes. The Journal of Forensic Psychiatry \& Psychology, v. 26, n. 1, p. 60-77, 2015.

61 BORRANI, Jorge et al. Analysis of Cognitive inhibition and flexibility in juvenile delinquentes. The Journal of Forensic Psychiatry \& Psychology, v. 26, n. 1, p. 60-77, 2015.

62 MOFFITT, Terrie; CASPI, Avshalom. Evidence from Behavioral Genetics for Environmental Contributions to Antisocial Conduct. In: WIKSTROM, Per-Olof; SAMPSON, Robert. Handbook of socialization: Theory and research. New York, NY, US: Guilford Press, 2007. p. 96-123.

63 URBINA, Susana. Fundamentos da testagem psicológica. Porto Alegre: Artmed, 2006.

64 MIYAGUCHI, Koji; SHIRATAKI, Sadaaki. Executive functioning problems of juveline sex offenders with low leves of measured intelligence. Journal of Intellectual \& Developmental Disability, v. 39, n. 3, p. 253-260, 2014. 
memória de trabalho). Nos subtestes das escalas Weschler, o grupo de adolescentes com histórico de delitos sexuais também teve desempenho inferior nos subtestes que avaliam Memória de Trabalho e Velocidade de Processamento. Destaca-se que não foram identificadas diferenças de desempenho entre os adolescentes de QI normativo - com e sem histórico de delitos sexuais. Essas evidências corroboram as obtidas no estudo de Muscatello e colegas ${ }^{65}$, no qual também se verificou uma relação entre déficits executivos e funcionamento intelectual global prejudicado.

Miyaguchi e Shirataki ${ }^{66}$ apontam que é provável que os adolescentes com histórico de delitos sexuais e baixo QI tenham mais defasagens desenvolvimentais que os adolescentes com histórico de delitos sexuais e QI normativo, ou que esse último grupo tenha o impacto das defasagens diminuído em função do QI mais alto. Dado que as evidências desta pesquisa se centraram, especialmente, nos déficits em habilidades de controle de interferência e em memória de trabalho, construtos que têm relação indireta com as manifestações comportamentais da delinquência, depreende-se que esses déficits, associados ao baixo QI, atuem mais no sentido de compor um quadro de desvantagens psicossociais que dificultam que o adolescente desenvolva comportamentos pró-sociais.

Burton, Demuynck e Yoder ${ }^{67}$ também investigaram a relação entre o funcionamento executivo e o cometimento de delitos sexuais. Uma amostra de adolescentes infratores $(\mathrm{n}=165)$ apreendidos pelo cometimento de delitos sexuais foi avaliada ${ }^{68}$ pelo Behavior Rating Inventory of Executive Function - Self Report (BRIEF-SR), versão autoaplicável do questionário que avalia os prejuízos decorrentes dos déficits executivos; pelo Self Report Delinquency (SRD), questionário que investiga os delitos que a pessoa já cometeu ao longo da vida; e pelo Self Report Sexual Aggression Scale (SERSAS), um inventário que investiga os comportamentos sexuais agressivos/violentos cometidos ao longo da vida. Não foram encontradas correlações entre as escalas do BRIEF e os delitos não sexuais cometidos. No entanto, foram encontradas correlações moderadas e pequenas entre os domínios de Inibição Comportamental e de Habilidades de Solução de Problemas da BRIEF e os delitos de natureza sexual. A falta de inibição comportamental/autocontrole pode ter influência direta e/ou indireta no desenvolvimento de condutas delituosas, conforme já apontado. O déficit em habilidades de solução de problemas, por seu turno, especialmente em relação ao domínio de flexibilidade cognitiva, ao dificultar o desenvolvimento de estilos pró-sociais efetivos, para resolver as demandas pessoais e relacionais, aumenta o risco de o adolescente adotar estilos antissociais, o que lhe torna vulnerável ao envolvimento em práticas ilegais — violentas e criminalizadas.

Por fim, destaca-se o estudo de Vilà-Balló, Hdez-Lafuente, Rostan, Cunillera e Rodriguez-Fornells ${ }^{69}$, no qual se empregou uma metodologia diferente daquela utilizada nas outras investigações para a aferição das FEs apresentadas neste artigo. Os autores estudaram a atividade eletrofisiológica correspondente aos déficits das FEs em adolescentes infratores, por meio da aferição de potenciais elétricos esperados frente a situações em que se processa um feedback negativo ou se verifica a necessidade de inibir o próprio comportamento. Com isso, verificaram que os adolescentes violentos tiveram mais dificuldade nas tarefas que exigiam detecção de erros e inibição do próprio comportamento, comparados a adolescentes infratores não violentos. Esses resultados corroboram a tese de que dificuldades na captação de sinais sociais e na inibição comportamental aumentam as chances de se comportar de forma violenta em determinados contextos.

65 MUSCATELLO, Maria et al. Executive functions and basic symptoms in adolescent antisocial behavior: A cross-sectional study on an Italian sample of late-onset offenders. Comprehensive Psycbiatry, v. 55, p. 631-638, 2014.

66 MIYAGUCHI, Koji et al. Cognitive training for delinquents within a residential service in Japan. Children and Youth Services Review, v. 34, n. 9, p. 1762-1768, 2012.

67 BURTON, David; DEMUYNCK, Sophia; YODER, Jamie. Executive Dysfunction predicts delinquency but not characteristic of sexual agression among adolescent sexual offenders. Sexual Abuse: A Journal of Research and Treatment, v. 28, n. 8, p. 707-721, 2016.

68 Os instrumentos utilizados neste estudo são de autorrelato; contém perguntas referentes a comportamentos passados, frente as quais o respondente deve declarar sua ocorrência e/ou frequência.

69 VILÀ-BALLÓ, Adrià et al. Neurophysiological correlates of cognitive flexibility and feedback processing in violent juvenile offenders. Brain Research, v. 1610, p. 98-109, 2015. 
Em suma, o conjunto dos resultados das investigações relativas às FEs indica que elas, embora possam não ser a causa do delito, explicam algumas variações no comportamento individual. Assim, as investigações nesse campo são relevantes - em especial as que ponderam sobre as condições em que as FEs contam para o desenvolvimento do comportamento social. As evidências mais consistentes, advindas de estudos empregando diferentes metodologias/instrumentos, revelam que o domínio mais vezes associado ao comportamento delituoso é o da autorregulação / do autocontrole. Ao lado das outras funções cognitivas, a autorregulação / o autocontrole é uma das variáveis pessoais, talvez a de maior peso, relacionadas à resiliência mediante a exposição dos indivíduos a fatores de risco ambientais específicos para a delinquência ${ }^{70}$. Dessa forma, indivíduos com capacidades elevadas de autorregulação são menos suscetíveis às influências exercidas pelos pares de idade e menos propensos a agirem de forma impulsiva, conseguindo exercer maior controle sobre as contingências contextuais. Além disso, conseguem mais facilmente engajarem-se em atividades pró-sociais e serem bem-sucedidos nelas, o que gera experiências positivas que reforçam a opção por um estilo de vida pró-social, o que suscita um ciclo positivo de comportamentos socialmente ajustados e vivências reforçadoras. Ademais, embora os estudos não sejam homogêneos quanto aos déficits encontrados nas FEs, em infratores, os dados levam a crer que a dificuldade de regular o comportamento em função da sensibilidade diferencial à recompensa e à punição é muito relevante no desenvolvimento do comportamento delituoso ${ }^{71}$.

Tais constatações podem ter importantes implicações em intervenções com adolescentes em conflito com a lei. Com o objetivo de explorar as possibilidades já aventadas nesse campo, expõem-se a seguir, brevemente, as abordagens e as técnicas atinentes às FEs, usualmente empregadas em programas de prevenção e de tratamento concernentes ao envolvimento de adolescentes com prática de delitos.

\section{IMPLICAÇÕES DO CONHECIMENTO SOBRE AS FES EM INTERVENÇÕES EM DELINQUÊNCIA JUVENIL}

A neuroplasticidade na adolescência atinge níveis que não se repetirão nas fases posteriores da vida ${ }^{72}$, o que torna os adolescentes potencialmente capazes de se adaptarem às mais diversas situações. $\mathrm{O}$ sentido do termo "adaptação", utilizado nesta seção deste artigo, não necessariamente representa "a forma ideal de enfrentar um determinado problema"; trata-se mais propriamente do processo de ajuste do indivíduo ao meio em que está inserido e dos resultados que decorrem, podendo ser um ajuste pró-social e/ou saudável ou antissocial e/ou patológico. Em outras palavras, a adolescência, por ser um período de alta suscetibilidade aos estímulos ambientais, é, em si mesmo, um momento de vulnerabilidade para o surgimento de problemas de comportamento e até mesmo de transtornos mentais, em face à adaptação que se processa a ambientes precários, violentos, hostis e/ou criminalizados.

Os estudos estimam que um em cada cinco adolescentes tem algum transtorno mental que persistirá na vida adulta, como transtornos de humor, de controle de impulsos, de uso de substâncias e de conduta ${ }^{73}$

\footnotetext{
70 MOFFITT, Terrie et al. A gradient of childhood self-control predicts health, wealth, and public safety. Proceedings of the National Academy of Sciences, v. 108, n. 7, p. 2693-2698, 2011.

71 SYNGELAKI, Eva et al. Executive Functioning and risky decision making in Young male offenders. Criminal Justice and Behavior, v. 36, n. 11, p. 1213-1227, 2009.

72 LEE, Francis et al. Adolescent mental health-Opportunity and obligation: Emerging neuroscience offers hope for treatments. Science, New York, v. 346, n. 6209, p. 547-549, 2014.

73 KAUFMANN, Tobias et al. Delayed stabilization and individualization in connectome development are related to psychiatric disorders. Nature Neuroscience, v. 20, n. 4, p. 513-515, 2017.
} 
74 75. Um relatório da Organização Mundial de Saúde ${ }^{76}$ revela que os gastos relacionados ao tratamento de transtornos mentais que surgem antes da vida adulta são cerca de 10 vezes maiores que os relacionados a transtornos que surgem durante a vida adulta. Assim, em uma primeira instância, é de suma importância evitar o surgimento dessas condições ou detectá-las precocemente de modo a aumentar as chances de sucesso da intervenção ${ }^{77}{ }^{78}$.

Com esse intuito, descrevem-se modalidades de ações, baseadas em evidências, que têm como objetivo promover o desenvolvimento das FEs e, assim, contribuir para a proteção da população infanto-juvenil contra uma série de problemáticas de ordem afetiva, cognitiva e comportamental. Essas ações implicam modificações ambientais e podem ser categorizadas em dois eixos: 1. Prevenção em âmbito familiar e escolar; 2. Tratamento em contextos específicos. A primeira ação é de cunho preventivo e prevê atuações nos contextos em que as crianças e os adolescentes passam a maior parte de seu tempo: em casa e na escola. É consenso entre os pesquisadores que políticas de prevenção são as medidas de melhor custo-benefício para a problemática da delinquência juvenil. ${ }^{79} \mathrm{~A}$ segunda é de caráter interventivo, e se faz necessária quando a prevenção falhou e os problemas decorrentes das defasagens/dos déficits desenvolvimentais aparecem; estas são, porém, mais custosas e requerem a atuação de profissionais mais especializados.

A prevenção em âmbito familiar deve ocorrer, primariamente, por meio dos adultos responsáveis pela socialização da criança. O Centro da Criança em Desenvolvimento da Universidade de Harvard ${ }^{80}$ disponibiliza na internet um guia gratuito de atividades que estimulam as FEs em crianças e adolescentes que podem ser incorporados às práticas parentais. Essas atividades foram desenvolvidas com base em estudos científicos, cujos detalhes são especificados em cada um dos guias disponíveis em sua página na internet. As atividades variam de estimulações sensoriais em bebês a jogos de imaginação, interpretação e de contar histórias na infância e a jogos de cartas e de tabuleiros que envolvem raciocínios lógicos e abstratos para adolescentes. Complementarmente, Galisnky ${ }^{81}$, em seu livro, aborda sete habilidades de vida essenciais a toda criança: foco e autocontrole; pensamento em perspectiva; comunicação; construir conexões; pensamento crítico; enfrentamento de problemas; aprendizado autônomo e engajado. O livro possui linguagem simples e serve como um guia instrutivo para pais/responsáveis que desejam estimular essas habilidades em seus filhos.

Um ponto crítico da ação preventiva com foco nas FEs refere-se ao fato de que não se tem a situação ideal em que todos os pais/responsáveis buscam por livre e espontânea vontade se informar e se formar a respeito dessas práticas estimuladoras e protetivas para o desenvolvimento da criança e do adolescente. Nesse sentido, uma medida conveniente seria estimular seu interesse pela temática por meio da confecção e do compartilhamento de cartilhas e de vídeos informativos, mostrando o que são, qual a importância e como é possível estimular as FEs. Uma segunda medida seria preparar profissionais da educação e da saúde, como os dos programas de Saúde da Família, para instruírem e incentivarem os cuidadores — pais/responsáveis — a respeito das FEs e das práticas educativas que podem adotar para estimulá-las ${ }^{82}$.

74 LEE, Francis et al. Adolescent mental health-Opportunity and obligation: Emerging neuroscience offers hope for treatments. Science, New York, v. 346, n. 6209, p. 547-549, 2014.

75 PAUS, Tomás; KESHAVAN, Matcheri; GIEDD, Jay. Why do many psychiatric disorders emerge during adolescence? Nature Reviews Neuroscience, v. 9, n. 12, p. 947-957, 2008.

76 ORGANIZAÇÃO MUNDIAL DA SAÚDE (OMS). Economic Aspects of Mental Health in Children and Adolescents. Geneva: WHO, 2007.

77 BEECHAM, Jennifer. Annual Research Review: Child and adolescent mental health interventions: A review of progress in economic studies across different disorders. Journal of Child Psychology and Psychiatry, v. 55, n. 6, p. 714-732, 2014.

78 LOEBER, Rolf; FARRINGTON, David; WASCHBUSCH, Daniel. Serious and Violent Offenders. In: LOEBER, Ralf; FARRINGTON, Davis. (Ed.) Serious and violent juvenile offenders: risk factors and successful interventions. Thousand Oaks, CA: Sage, 1998. 79 LOEBER, Rolf; FARRINGTON, David; WASCHBUSCH, Daniel. Serious and Violent Offenders. In: LOEBER, Ralf; FARRINGTON, Davis. (Ed.) Serious and violent jwvenile offenders: risk factors and successful interventions. Thousand Oaks, CA: Sage, 1998. 80 CENTER ON THE DEVELOPING CHILD AT HARVARD UNIVERSITY. Enhancing and practicing executive function skills with children from infancy to adolescence. 2014.

81 GALINSKY, Ellen. Mind in the making: the seven essential life skills every child needs. Harper Collins E-books, 2010.

82 MEUWISSEN, Alyssa; ENGLUND, Michelle. Executive function in at-risck children: importance of father-figure support 
Outro espaço estratégico para promover práticas preventivas é diretamente na escola. Segundo Meltzer e Basho ${ }^{83}$, é possível criar ambientes escolares em que os processos das FEs sejam focalizados e possam ser sistematicamente estimulados, compondo, assim, um tema transversal nos currículos. Com base em resultados do Instituto de Pesquisa para Aprendizagem e Desenvolvimento e revisões metanalíticas da literatura, as autoras descrevem um programa para instrumentalizar os profissionais da educação que desejem implantar esse tipo de "cultura" em sala de aula ou na escola. A ideia é que os profissionais consigam adaptar os princípios gerais da implementação da estimulação das FEs em ambiente escolar ao contexto de suas próprias escolas, começando pela avaliação das capacidades executivas de cada aluno. Sugerem que, a partir disso, se pode estabelecer uma estratégia de desenvolvimento de FEs essenciais ao aprendizado e ao sucesso acadêmico como o estabelecimento, o planejamento e a priorização de tarefas ${ }^{84}$; a manipulação mental de informações ${ }^{85}$; a flexibilidade cognitiva para solução de problemas $^{86}$; o automonitoramento e a autorregulação emocional ${ }^{87}$ ${ }^{88}$. Essas aquisições não apenas protegem o aluno de fracassar e evadir a escola como também podem ser generalizadas a outros contextos, formando indivíduos resilientes a uma série de problemáticas da vida.

A segunda modalidade de atuação remete às intervenções necessárias quando o adolescente já apresenta algum prejuízo socioemocional, que pode ser decorrente de alguma disfunção executiva. Refere-se, assim, a intervenções de prevenção secundária ou de tratamento. Em alguns casos, sem desconsiderar aspectos sociais e contextuais relevantes da vida do indivíduo, a defasagem desenvolvimental pode ter se acumulado de modo a participar como fator relevante à implicação do adolescente com a prática de delitos. Nessa situação, o primeiro procedimento é a realização de uma avaliação fundada em instrumentos confiáveis e a validação ecológica dos resultados por meio da verificação de sua compatibilidade com o que se observa na vida real do adolescente ${ }^{89}$. Substanciado pela avaliação, um plano interventivo deve ser elaborado com o objetivo de suprir as necessidades desenvolvimentais do adolescente, devendo este ser, ao mesmo tempo, compatível com suas capacidades e com o seu contexto de vida.

No caso dos adolescentes em conflito com a lei, tais condições são previstas pelo Estatuto da Criança e do Adolescente ${ }^{90}$ e pelo Sistema Nacional de Atendimento Socioeducativo ${ }^{91}$. Todavia, o Estatuto da Criança e do Adolescente (ECA), no seu artigo 112, prescreve que "A medida aplicada ao adolescente levará em conta a sua capacidade de cumpri-la, as circunstâncias e a gravidade da infração", ante a falta de fundamentação científica apropriada, tem gerado práticas no âmbito de todo o sistema de justiça juvenil que riscam se nortear por critérios totalmente subjetivos (para não dizer discricionários), ou que se assentam quase que exclusivamente na apreciação das "circunstâncias e da gravidade da infração", à luz de critérios jurídicos".

and mother parenting. Journal of Applied Developmental Psychology, v. 44, p. 72-80, 2016.

83 MELTZER, Lynn; BASHO, Surina. Creating a Classroomwide Executive Function Culture That Fosters Strategy Use, Motivation, and Resilience. In: MELTZER, Lynn (Ed.). Promotin executive functions in the classroom. New York: Guildford Press, 2010. p. 28-52.

84 KRISHNAN, Kalyani; FELLER, Melissa; ORKIN, Melissa. Goal Setting, Planning, and Prioritizing: The Foundations of Effective Learning. In: MELTZER, Lynn (Ed.). Promotin executive functions in the classroom. New York: Guildford Press, 2010. p. 57-85. 85 KINCAID, Donna; TRAUTMAN, Nancy. Remembering: Teaching Students How to Retain and Mentally Manipulate Information. In: MELTZER, Lynn (Ed.). Promotin executive functions in the classroom. New York: Guildford Press, 2010. p. 110-139.

86 MELTZER, Lynn; BAGNATO, Jennifer. Shifting and Flexible Problem Solving: The Anchors for Academic Success. In: MELTZER, Lynn (Ed.). Promotin executive functions in the classroom. New York: Guildford Press, 2010. p. 140-159.

87 BAGNATO, Jennifer; MELTZER, Lynn. Self- Monitoring and Self- Checking: The Cornerstones of Independent Learning. In: MELTZER, Lynn (Ed.). Promotin executive functions in the classroom. New York: Guildford Press, 2010. p. 160-173.

88 STEIN, Judith. Emotional Self- Regulation: A Critical Component of Executive Function. In: MELTZER, Lynn (Ed.). Promotin executive functions in the classroom. New York: Guildford Press, 2010. p. 175-201.

89 BARKLEY, Russell. Executive functions: what they are, how they work, and why they evolve. New York: The Guilford Press, 2012.

90 BRASIL. Estatuto da criança e do adolescente. São Paulo: Atlas, 1991.

91 BRASIL. Lei n. 12.594, de 18 de janeiro de 2012. Disponível em: <http://www.planalto.gov.br/ccivil_03/_ato2011-2014/2012/ lei/112594.htm>. Acesso em: 17 abr. 2018.

92 MARUSCHI, Maria Cristina; BAZON, Marina. Justiça Juvenil: a aplicação e a execução das medidas socioeducativas pelos parâmetros do modelo "risco-necessidade-responsividade". Rio de Janeiro: Instituto INNOVARE, 2014. v. 1. p. 42-72. 
Conforme indicam Komatsu e Bazon ${ }^{93}$, no contexto sociocultural brasileiro, ainda, se padece da falta de referenciais teóricos e metodológicos consistentes, e parâmetros confiáveis para a devida interpretação do que seria a "capacidade de cumprir" a medida.

Na perspectiva da neurociência, o estado de funcionamento do próprio indivíduo deveria ser considerado enquanto parte das circunstâncias e da gravidade da infração. Conforme se argumentou anteriormente, muitas crianças e adolescentes não são capazes de atingir um nível de autorregulação comportamental necessário para evitar infringir as regras ou as leis em face às condições ambientais em que estão inseridos. Isto não significa ser complacente com todo e qualquer comportamento dos jovens sob a justificativa de que eles não são ainda capazes de fazer diferente. Ao contrário, implica elaborar e propor respostas jurídicas e socais contingentes e consistentes frente aos comportamentos indesejáveis.

McCloskey, Perkins e Van Diviner ${ }^{94}$ salientam que é muito difícil para algumas figuras de autoridade aceitar que as consequências que devem ser aplicadas aos comportamentos indesejáveis de um jovem sejam outras que a punição. Para os autores, essa dificuldade baseia-se na crença de que não punir comportamentos errados "ensina" os jovens que eles podem fazer o que quiserem; ou que os jovens obedientes se sentiriam injustiçados vendo outros fazerem coisas erradas sem serem punidos. Nesse sentido, uma consequência punitiva suscita um sentimento de justiça no agente da punição ou na sociedade ${ }^{95}$; mas, em muitos casos, ela não é capaz de gerar o comportamento desejável no indivíduo punido ${ }^{96}{ }^{97}$. Nessas situações, a solução seria promover competências no infrator para que ele consiga se comportar de forma apropriada e ter a oportunidade de ser reforçado positivamente por isso. É com base nessa lógica que surge a necessidade de intervenções diferenciadas focalizadas nas necessidades de autoregulação de cada indivíduo.

Para McCloskey, Perkins e Van Diviner ${ }^{98}$, a intervenção em relação às dificuldades executivas tem três premissas básicas: 1. as dificuldades nas funções executivas estão relacionadas a um sub funcionamento do cérebro; 2. as funções cerebrais podem ser alteradas por meio de intervenções; 3. as intervenções podem ativar funções intactas do cérebro. Dessa forma, considera-se que a plasticidade cerebral - capacidade do cérebro em mudar sua organização e seu funcionamento com base em atividades e experiências anteriores - é a base sobre a qual se propõe um treinamento/uma intervenção de prevenção secundária (ou tratamento) $)^{99} 100$.

A ideia básica de uma intervenção dessa natureza é a de que os indivíduos podem melhorar suas performances se experimentarem condições que ativem repetidamente regiões neurais envolvidas na execução de tarefas que requerem a função de interesse, por meio de um treinamento regular, durante um período suficientemente extenso ${ }^{101} 102$. Como consequência, o efeito do treinamento pode se generalizar para outras

93 KOMATSU, André Vilela; BAZON, Marina Rezende. Adolescentes em conflito com a lei: justiça juvenil pela perspectiva da criminologia desenvolvimental. In: NOJIRI, S. (Org.). Direito, Psicologia e Neurociência. Ribeirão preto: IELD, 2016. 186p.

94 MCCLOSKEY, George; PERKINS, Lisa; VAN DIVINER, Bob. Assessment and Intervention for Executive Function Difficulties. New York: Routledge, 2009.

95 Dias e Custódio oferecem uma crítica sobre como o discurso punitivo relacionado a crianças e adolescentes não só está enraizado no ideário popular, como também afeta a agenda política brasileira: DIAS, Felipe da Veiga; CUSTÓDIO, André Viana. O discurso expansivo-punitivo dos meios de comunicação e sua influência na formação da agenda das políticas públicas de combate à criminalidade de crianças e de adolescentes no Brasil. Rev. Bras. Polít. Públicas, Brasília, v. 3, n. 1, p. 91-104, 2013.

96 GERSHOFF, Elizabeth. Corporal punishment by parents and associated child behavior and experiences: A meta-analysis and theoretical review. Psychological Bulletin, v. 128, p. 539-579, 2002.

97 SKINNER, Burrhus Frederic. About Behaviorism. New York: Knopf, 1974.

98 MCCLOSKEY, George; PERKINS, Lisa; VAN DIVINER, Bob. Assessment and Intervention for Executive Function Difficulties. New York: Routledge, 2009.

99 DIAMOND, Adele; LING, Daphne. Conclusions about interventions, programs, and approaches for improving executive functions that appear justified and those that, despite much hype, do not. Developmental Cognitive Neuroscience, v. 18, p. 34-48, 2016.

100 ROMERO, María. Executive control enhancement and cognitive training. 2017. Tese (Doutorado), Universidad de Granada, Granada, 2017.

101 ROMERO, María. Executive control enhancement and cognitive training. 2017. Tese (Doutorado) - Universidad de Granada, Granada, 2017.

102 STOKES, Mark; BUSCHMAN, Timothy; MILLER, Earl. Dynamic Coding for Flexible Cognitive Control. The Wiley Hand- 
tarefas que não foram treinadas, mas que fazem parte do mesmo domínio funcional ${ }^{103}$. A noção do efeito generalizador da estimulação de circuitos neurais foi testada em uma série de intervenções e os resultados mostraram efeitos positivos 104105106107.

De maneira geral, os programas de intervenção indicam exercitar as mesmas áreas que os programas de prevenção; a diferença subjaz na importância de o tratamento focar nas necessidades pessoais específicas, identificadas como defasadas/deficitárias no beneficiário da intervenção. Com base em estudos experimentais, inúmeros programas se mostraram efetivos em melhorar a performance das FEs: jogos computadorizados que exercitam a memória de trabalho (aplicativos com esse tipo de jogo possuem versões gratuitas na internet ${ }^{108}$; jogos de tabuleiros que estimulam o raciocínio lógico ${ }^{109}$; exercícios aeróbicos e a prática esportiva, desde que associados a algum treinamento cognitivo ${ }^{110}{ }^{111}$; prática de Yoga e de Mindfullness ${ }^{112}{ }^{113}$; exercícios cognitivos de papel e caneta ${ }^{114}$; psicoterapia cognitivas e comportamentais ${ }^{115}$.

Contudo, as evidências mostram que a estimulação das FEs não é igualmente efetiva para todas as pessoas, não ficando claro nos estudos por que algumas pessoas se beneficiam mais que outras. Isso tem implicações importantes na eficácia da intervenção individual no mundo real. A esse respeito, Diamond e Ling ${ }^{116}$ argumentam que há, certamente, outros fatores influenciando nesse processo. Os autores indicam que, sendo as FEs reguladas, principalmente, pelo córtex pré-frontal (CPF), a área evolutivamente mais jovem do cérebro, elas são muito vulneráveis a situações de estresse, tristeza, solidão e má condição física. Sabe-se que as tarefas que exigem raciocínio, autocontrole e adaptação à mudança são melhor desempenhadas quando o indivíduo está menos estressado, quando se sente socialmente e emocionalmente acolhido e quando está bem de saúde (incluindo qualidade do sono). Por conseguinte, Diamond e Ling ${ }^{117}$ hipotetizam que as intervenções mais bem-sucedidas são aquelas que estimulam não somente as FEs, diretamente, mas sim as que incluem também estratégias que indiretamente reduzem os fatores que impactam, negativamente, o CPF e promovem os fatores que impactam, positivamente, o CPF. Todos esses fatores deveriam ser levados em consideração na determinação e na execução das medidas socioeducativas aos adolescentes em conflito com a lei.

book of Cognitive Control, p. 221-241, 2017.

103 ROMERO, María. Executive control enhancement and cognitive training. 2017. Tese (Doutorado) - Universidad de Granada, Granada, 2017.

104 DIAMOND, Adele. Executive functions. Annual Review of Psychology, v. 64, p. 135-168, 2013.

105 DIAMOND, Adele; LING, Daphne. Conclusions about interventions, programs, and approaches for improving executive functions that appear justified and those that, despite much hype, do not. Developmental Cognitive Neuroscience, v. 18, p. 34-48, 2016.

106 ENRIQUEZ-GEPPERT, Stefanie; HUSTER, René; HERRMANN, Christoph. Boosting brain functions: Improving executive functions with behavioral training, neurostimulation, and neurofeedback. International Journal of Psychophysiology, v. 88, n. 1, p. 1-16, 2013.

107 MCCLOSKEY, George; PERKINS, Lisa; VAN DIVINER, Bob. Assessment and Intervention for Executive Function Difficulties. New York: Routledge, 2009.

108 DIAMOND, Adele. Executive functions. Annual Review of Psychology, v. 64, p. 135-168, 2013.

109 DIAMOND, Adele; LEE, Kathleen. Interventions shown to Aid Executive Function Development in Children 4-12 Years Old. Science, New York, v. 333, n. 6045, p. 959-964, 2011.

110 DIAMOND, Adele. Executive functions. Annual Review of Psychology, v. 64, p. 135-168, 2013.

111 DIAMOND, Adele; LING, Daphne. Conclusions about interventions, programs, and approaches for improving executive functions that appear justified and those that, despite much hype, do not. Developmental Cognitive Neuroscience, v. 18, p. 34-48, 2016.

112 DIAMOND, Adele; LEE, Kathleen. Interventions shown to Aid Executive Function Development in Children 4-12 Years Old. Science, New York, v. 333, n. 6045, p. 959-964, 2011.

113 DIAMOND, Adele; LING, Daphne. Conclusions about interventions, programs, and approaches for improving executive functions that appear justified and those that, despite much hype, do not. Developmental Cognitive Neuroscience, v. 18, p. 34-48, 2016.

114 MIYAGUCHI, Koji et al. Cognitive training for delinquents within a residential service in Japan. Children and Youth Services Review, v. 34, n. 9, p. 1762-1768, 2012.

115 MCCLOSKEY, George; PERKINS, Lisa; VAN DIVINER, Bob. Assessment and Intervention for Executive Function Difficulties. New York: Routledge, 2009.

116 DIAMOND, Adele; LING, Daphne. Conclusions about interventions, programs, and approaches for improving executive functions that appear justified and those that, despite much hype, do not. Developmental Cognitive Neuroscience, v. 18, p. 34-48, 2016. 117 DIAMOND, Adele; LING, Daphne. Conclusions about interventions, programs, and approaches for improving executive functions that appear justified and those that, despite much hype, do not. Developmental Cognitive Neuroscience, v. 18, p. 34-48, 2016. 


\section{Considerações Finais}

A adolescência é um período de grandes transformações neurodesenvolvimentais. Pela plasticidade que lhe é inerente, representa, ao mesmo tempo, um recurso e uma vulnerabilidade. As transformações em curso apresentam-se como um "período sensível", ou seja, um período em que a aprendizagem de habilidades ou desenvolvimento de aptidões e competências se faz de modo intenso e mais fácil. Assim, de um lado, se os estímulos ambientais existem e são adequados, o adolescente faz aquisições que lhe permitirá lidar de modo cada vez mais competente com as complexas demandas psicossociais. De outro lado, se os estímulos ambientais não existem ou são inadequados, haverá defasagens/déficits que perpassarão sua adaptação psicossocial, concorrendo para a emergência de inúmeros problemas de comportamento e, até mesmo, transtornos mentais. Sem a estimulação adequada, o desenvolvimento dos circuitos neurais pode sofrer um atraso, limitando as capacidades executivas do adolescente e aumentando sua dificuldade em responder apropriadamente às demandas sociais.

Em relação a essa perspectiva, no presente artigo buscou-se integrar os conhecimentos científicos acerca das principais FEs relacionadas ao comportamento delituoso, no escopo do desenvolvimento do adolescente. As FEs são importantes para o funcionamento adaptativo em praticamente todas as esferas da vida. Defasagens/déficits nas FEs podem se relacionar com a prática delituosa de forma indireta e direta. $\mathrm{Na}$ forma indireta, disfunções executivas contribuem para a existência de dificuldades de ajustamento ao meio acadêmico e também ao laboral, que aumentam as chances de o adolescente se afastar dessas instituições sociais, de regulação da conduta e, assim, se envolver com práticas divergentes e/ou delituosas. $\mathrm{Na}$ forma direta, disfunções executivas, especialmente no plano do controle de impulsos, contribuem para a emissão de delitos específicos e para a violência.

A literatura sugere que as FEs podem ser estimuladas e desenvolvidas. Assim, buscou-se igualmente pontuar ações específicas para diminuir os fatores de risco e promover os fatores de proteção em relação ao desenvolvimento pleno das FEs. As ações preventivas podem ser aplicadas desde os primeiros meses de vida até à adolescência e possuem como foco os contextos familiar e escolar, em que as crianças passam a maior parte de seu tempo. As ações de tratamento (ou de prevenção secundária) devem ser mais específicas, no sentido de focalizar as FEs mais diretamente relacionadas aos problemas manifestos, e mais duradouras, além de levar em consideração fatores contraproducentes ao desenvolvimento das FEs, como o estresse, o isolamento social/emocional e a saúde como um todo.

Por último, é preciso destacar que, a despeito da velocidade com a qual as ciências relacionadas ao desenvolvimento humano estão progredindo, com o advento de novas tecnologias e métodos de investigação, oferecendo muito conhecimento útil, pouco ou quase nada foi incorporado às legislações e às políticas públicas brasileiras. Assim, o desafio imediato consiste em envidar esforços para que haja maior difusão e integração desse tipo de conhecimento.

\section{REFERÊNCIAS}

ARAIN, Mariam et al. Maturation of the adolescent brain. Neuropsychiatric Disease and Treatment, v. 9, p. 449461, 2013.

BADDELEY, Alan. Working memory. Science, v. 255, n. 5044, p. 556-9, 1992.

BAGNATO, Jennifer; MELTZER, Lynn. Self- Monitoring and Self- Checking: The Cornerstones of Independent Learning. In: MELTZER, Lynn (Ed.). Promotin executive functions in the classroom. New York: Guildford Press, 2010. p. 160-173. 
BARKLEY, Russell. Executive functions: what they are, how they work, and why they evolve. New York: The Guilford Press, 2012.

BAZON, Marina; SILVA, Jorge; FERRARI, Renata. Trajetórias escolares de adolescents em conflito com a lei. Educação em Revista, Belo Horizonte, v. 29, n. 02, p. 175-199. 2013.

BEECHAM, Jennifer. Annual research review: child and adolescent mental health interventions: a review of progress in economic studies across different disorders. Journal of Child Psychology and Psychiatry, v. 55, n. 6, p. 714-732, 2014.

BORRANI, Jorge et al. Analysis of Cognitive inhibition and flexibility in juvenile delinquentes. The Journal of Forensic Psychiatry \& Psychology, v. 26, n. 1, p. 60-77, 2015.

BRASIL. Estatuto da criança e do adolescente. São Paulo: Atlas, 1991.

BRASIL. Lei n. 12.594, de 18 de janeiro de 2012. Disponível em: <http://www.planalto.gov.br/ccivil_03/_ ato2011-2014/2012/lei/112594.htm>. Acesso em: 17 abr. 2018.

BURTON, David; DEMUYNCK, Sophia; YODER, Jamie. Executive Dysfunction predicts delinquency but not characteristic of sexual agression among adolescent sexual offenders. Sexual Abuse: A Journal of Research and Treatment, v. 28, n. 8, p. 707-721, 2016

CENTER ON THE DEVELOPING CHILD AT HARVARD UNIVERSITY. Enhancing and practicing executive function skills with children from infancy to adolescence. 2014. Disponível em: <https://children.wi.gov/Documents/Harvard\%20Parenting\%20Resource.pdf>. Acesso em: 18 abr. 2018.

DAJANI, Dina; UDDIN, Lucina. Demystifying cognitive flexibility: Implications for clinical and developmental neuroscience. Trends in Neurosciences, v. 38, n. 9, p. 571-578, 2015.

DELISI, Matt. Low Self-Control Is a Brain-Based Disorder. In: BEAVER, Kevin; BARNES, JC; BOUTWELL, Brian. The nurture versus biosocial debate in criminology: on the origins of criminal behavior and criminality. Thousand Oaks: Sage Publication Inc., 2014. p. 172-183.

DIAMOND, Adele. Executive functions. Annual Review of Psychology, v. 64, p. 135-168, 2013.

DIAMOND, Adele; LEE, Kathleen. Interventions shown to aid executive function development in children 4-12 years old. Science, New York, v. 333, n. 6045, p. 959-964, 2011.

DIAMOND, Adele; LING, Daphne. Conclusions about interventions, programs, and approaches for improving executive functions that appear justified and those that, despite much hype, do not. Developmental Cognitive Neuroscience, v. 18, p. 34-48, 2016.

DIAS, Felipe da Veiga; CUSTÓDIO, André Viana. O discurso expansivo-punitivo dos meios de comunicação e sua influência na formação da agenda das políticas públicas de combate à criminalidade de crianças e de adolescentes no Brasil. Rev. Bras. Polít. Públicas, Brasília, v. 3, n. 1, p. 91-104, 2013.

DUCKWORTH, Angela; KERN, Margaret. A meta-analysis of the convergent validity of self-control measures. Journal of Research in Personality, v. 45, n. 3, p. 259-268, 2011.

ENNS, Richard et al. Measuring executive functions in female delinquents using the Cognitive assessment system. Journal of Offender Rehabilitation, v. 47, n. 1/2, p. 3-23, 2008.

ENRIQUEZ-GEPPERT, Stefanie; HUSTER, René; HERRMANN, Christoph. Boosting brain functions: Improving executive functions with behavioral training, neurostimulation, and neurofeedback. International Journal of Psychophysiology, v. 88, n. 1, p. 1-16, 2013.

FARRINGTON, David et al. Criminal careers up to age 50 and life success up to age 48: new findings from the cambridge study in delinquent development. PsycEXTRA Dataset, 2006. 
FINN, Emily et al. Functional connectome fingerprinting: identifying individuals using patterns of brain connectivity. Nature Neuroscience, v. 18, n. 11, p. 1664-1671, 2015.

FRANCO, Mariana; BAZON, Marina. Social information processing and aggravation of conduct in young offenders. International Annals of Criminology, v. 55, n. 01, p. 114-131, 2017.

FUJITA, Kentaro. On conceptualizing self-control as more than the effortful inhibition of impulses. Personality and Social Psychology Review, v. 15, n. 4, p. 352-366, 2011.

GALINSKY, Ellen. Mind in the making: the seven essential life skills every child needs. Harper Collins Ebooks, 2010.

GALVÁN, Adriana. Adolescence, brain maturation and mental health. Nature Neuroscience, v. 20, n. 4, p. 503-504, 2017.

GERSHOFF, Elizabeth. Corporal punishment by parents and associated child behavior and experiences: a meta-analysis and theoretical review. Psychological Bulletin, v. 128, p. 539-579, 2002.

GOTTFREDSON, Michael; HIRSCHI, Travis. A general theory of crime. Stanford, CA: Stanford University Press, 1990.

GREENWOOD, Pamela. Functional plasticity in cognitive aging: review and hypothesis. Neuropsychology, v. 21, n. 6, p. 657-673, 2007.

HAUSER, Tobias et al. Cognitive flexibility in adolescence: Neural and behavioral mechanisms of reward prediction error processing in adaptive decision making during development. Neuroimage, v. 104, p. 347-354, 2015.

HUGHES, Claire; GRAHAM, Andrew; GRAYSON, Andrew. Executive function in childhood: Development and disorder. In: OATES, John. (Ed.). Cognitive Development. Oxford: Open University Press, p. 2005. 205-230.

JEVTIC, Bisera. Moral Judgement of Delinquents. Procedia: Social and Behavioral Sciences, v. 149, p. 449455, 2014.

JOYAL, Christian; BEAULIEU-PLANTE, Jolyane; CHANTÉRAC, Antoine. The neuropsychology of sex offenders: a meta analysis. Sexual Abuse: A Jornal of Research and Treatment, v. 20, n. 10, p. 1-29, 2013.

KAUFMANN, Tobias et al. Delayed stabilization and individualization in connectome development are related to psychiatric disorders. Nature Neuroscience, v. 20, n. 4, p. 513-515, 2017.

KINCAID, Donna; TRAUTMAN, Nancy. Remembering: Teaching Students How to Retain and Mentally Manipulate Information. In: MELTZER, Lynn (Ed.). Promotin executive functions in the classroom. New York: Guildford Press, 2010. p. 110-139.

KOMATSU, André Vilela; BAZON, Marina Rezende. Adolescentes em conflito com a lei: justiça juvenil pela perspectiva da criminologia desenvolvimental. In: NOJIRI, S. (Org.). Direito, Psicologia e Neurociência. Ribeirão preto: IELD, 2016. 186p.

KOMATSU, André Vilela; BAZON, Marina Rezende. Personal differences among brazilian adolescents with distinct levels of engagement in delinquency. International Journal of Criminology and Sociology, v. 6, p. 6574, 2017.

KONRAD, Kerstin; FIRK, Christine; UHLHAAS, Peter. Brain development during adolescence: neuroscientific insights into this developmental period. Deutsches Ärateblatt International, v. 110, n. 25, p. 425-431, 2013.

KRISHNAN, Kalyani; FELLER, Melissa; ORKIN, Melissa. Goal setting, planning, and prioritizing: the foundations of effective learning. In: MELTZER, Lynn (Ed.). Promotin executive functions in the classroom. New 
York: Guildford Press, 2010. p. 57-85.

LEE, Francis et al. Adolescent mental health: opportunity and obligation: Emerging neuroscience offers hope for treatments. Science, New York, v. 346, n. 6209, p. 547-549, 2014.

LIMA, Renata Mantovani de; POLI, Leonardo Macedo; JOSÉ, Fernanda São. A evolução histórica dos direitos da criança e do adolescente: da insignificância jurídica e social ao reconhecimento de direitos e garantias fundamentais. Rev. Bras. Polít. Públicas, Brasília, v. 7, n. 2, p. 313-329, 2017.

LOEBER, Rolf; FARRINGTON, David; WASCHBUSCH, Daniel. Serious and violent offenders. In: LOEBER, Ralf; FARRINGTON, Davis. (Ed.) Serious and violent juvenile offenders: risk factors and successful interventions. Thousand Oaks, CA: Sage, 1998. p.13-29.

MARUSCHI, Maria Cristina; BAZON, Marina. Justiça Juvenil: a aplicação e a execução das medidas socioeducativas pelos parâmetros do modelo "risco-necessidade-responsividade". Rio de Janeiro: Instituto INNOVARE, 2014. v. 1. p. 42-72.

MCCLOSKEY, George; PERKINS, Lisa; VAN DIVINER, Bob. Assessment and intervention for executive function difficulties. New York: Routledge, 2009.

MEIJERS, Jesse et al. Differences in executive functioning between violent and non-violent offenders. Psychological Medicine, v. 47, n. 10, p. 1784-1793, 2017.

MELTZER, Lynn; BAGNATO, Jennifer. Shifting and Flexible Problem Solving: The Anchors for Academic Success. In: MELTZER, Lynn (Ed.). Promotin executive functions in the classroom. New York: Guildford Press, 2010. p. 140-159.

MELTZER, Lynn; BASHO, Surina. Creating a classroomwide executive function culture that fosters strategy use, motivation, and resilience. In: MELTZER, Lynn (Ed.). Promotin executive functions in the classroom. New York: Guildford Press, 2010. p. 28-52.

MEUWISSEN, Alyssa; ENGLUND, Michelle. Executive function in at-risck children: importance of father-figure support and mother parenting. Journal of Applied Developmental Psychology, v. 44, p. 72-80, 2016.

MILLER, Laurence. Neuropsychological perspectives on delinquency. Behavioral Sciences \& the Law, v. 6, p. 409-428, 1988.

MIYAGUCHI, Koji et al. Cognitive training for delinquents within a residential service in Japan. Children and Youth Services Review, v. 34, n. 9, p. 1762-1768, 2012.

MIYAGUCHI, Koji; SHIRATAKI, Sadaaki. Executive functioning problems of juveline sex offenders with low leves of measured intelligence. Journal of Intellectual \& Developmental Disability, v. 39, n. 3, p. 253-260, 2014.

MOFFITT, Terrie et al. A gradient of childhood self-control predicts health, wealth, and public safety. Proceedings of the National Academy of Sciences, v. 108, n. 7, p. 2693-2698, 2011.

MOFFITT, Terrie. Male antisocial behaviour in adolescence and beyond. Nature Human Behaviour, v. 2, n. 3 , p. 177-186, 2018.

MOFFITT, Terrie; CASPI, Avshalom. Evidence from Behavioral Genetics for Environmental Contributions to Antisocial Conduct. In: WIKSTROM, Per-Olof; SAMPSON, Robert. Handbook of socialization: Theory and research. New York, NY, US: Guilford Press, 2007. p. 96-123.

MUSCATELLO, Maria et al. Executive functions and basic symptoms in adolescent antisocial behavior: a cross-sectional study on an Italian sample of late-onset offenders. Comprehensive Psychiatry, v. 55, p. 631-638, 2014.

NORDVALL, Olov; JONSSON, Bert; NEELY, Anna. Self-reported and performance-based measures of executive functions in interned youth. Psychology, Crime \& Law, v. 23, n. 3, p. 240-253, 2016. 
NOREEN, Saima; MACLEOD, Malcolm. What do we really know about cognitive inhibition? task demands and inhibitory effects across a range of memory and behavioural tasks. PLOS ONE, v. 10, n. 8, 2015.

ORGANIZAÇÃO MUNDIAL DA SAÚDE. Economic Aspects of Mental Health in Children and Adolescents. Geneva: WHO, 2007.

PANWAR, Karni et al. Differential associations between impulsivity and risk-taking and brain activations underlying working memory in adolescents. Addictive Behaviors, v. 39, n. 11, p. 1606-1621, 2014.

PAUS, Tomás; KESHAVAN, Matcheri; GIEDD, Jay. Why do many psychiatric disorders emerge during adolescence? Nature Reviews Neuroscience, v. 9, n. 12, p. 947-957, 2008.

PIHET, Sandrine et al. Cognitive and emotional deficits associated with minor and serious delinquency in high-risk adolescents. Psychiatry, Psychology and Law, v. 19, n. 3, p. 427-438, 2012.

PONTIUS, Anneliese. Neurological aspects in some type of delinquency, especially among juveniles: toward a neurological model of ethical action. Adolescence, v. 7, p. 289-308, 1972.

ROMERO, María. Executive control enhancement and cognitive training. 2017. Tese (Doutorado), Universidad de Granada, Granada, 2017.

SKINNER, Burrhus Frederic. About Behaviorism. New York: Knopf, 1974.

STEIN, Judith. Emotional Self- Regulation: A Critical Component of Executive Function. In: MELTZER, Lynn (Ed.). Promotin executive functions in the classroom. New York: Guildford Press, 2010. p. 175-201.

STILES, Joan; JERNIGAN, Terry. The Basics of Brain Development. Neuropsychology Review, v. 20, n. 4, p. 327-348, 2010.

STOKES, Mark; BUSCHMAN, Timothy; MILLER, Earl. Dynamic Coding for Flexible Cognitive Control. The Wiley Handbook of Cognitive Control, p. 221-241, 2017.

STRAUSS, Esther; SHERMAN, Elisabeth; SPREEN, Otfried. A compendium of neuropsychological tests: administration, norms, and commentary. 3. ed. New York: Oxford University Press, 2016.

SYNGELAKI, Eva et al. Executive functioning and risky decision making in young male offenders. Criminal Justice and Behavior, v. 36, n. 11, p. 1213-1227, 2009.

URBINA, Susana. Fundamentos da testagem psicológica. Porto Alegre: Artmed, 2006.

VILÀ-BALLÓ, Adrià et al. Neurophysiological correlates of cognitive flexibility and feedback processing in violent juvenile offenders. Brain Research, v. 1610, p. 98-109, 2015.

VILÀ-BALLÓ, Adrià. Neuropshysiological correlates of error monitoring and inhibitory processing in juvenile violent offenders. Biological Psychology, v. 102, p. 141-152, 2014.

WALTERS, Glenn. Low self-control, peer rejection, reactive criminal thinking, and delinquent peer associations: connecting the pieces of the crime puzzle. Journal of Developmental and Life-Course Criminology, v. 2, n. 2, p. 209-231, 2016. 
Para publicar na revista Brasileira de Políticas Públicas, acesse o endereço eletrônico www.rbpp.uniceub.br

Observe as normas de publicação, para facilitar e agilizar o trabalho de edição. 